Warmblüter (Katze) tritt nach einiger Zeit eine bedeutende Vertiefung und Verlangsamung der Atmung ohne sonstige wesentliche Störungen ein; je nach der Grösse der Gabe kommt es zu Respirationsstillstand oder zu völliger Erholung. Die Zirkulation wird nur sekundär beeinflusst; keinerlei motorische oder sensible Lähmung. Beim Hund bewirkt das Anagyrin ein Erhöhung der Reflexerregbarkeit, doch kommt es nie za Krämpfen; sonstige Vergiftungserscheinungen wurden beim Hunde nicht bemerkt.

\title{
130. Ueber das Anagyrin.
}

Von Dr. F. M. Litterscheid.

Ueber Darstellungsweisen des Cytisins und Anagyrins aus den Samen der Anagyris foetida finden sich in der diesbezüglichen Litteratur Angaben von N. Reale, E. Hardy und Gallois, sowie ferner von Partheil und Spasski (l. c.). Von den dortselbst beschriebenen Verfahren konnte nur das von Partheil und Spasski ausgearbeitete in Frage kommen, weil dieses allein nicht nur eine Erschöpfung der Samen an den beiden Basen gewährleistet, sondern auch auf die exakte Trennung derselben Rücksicht nimmt und dabei vor den übrigen ausserdem noch den Vorzug der geringeren Umständlichkeit besitzt.

Partheil und Spasski sagen in ihrer vorläufigen Mitteilung:

„Behufs Darstellung der Alkaloide extrahierten wir die grob gepulverten Anagyrissamen in einem Perkolator mit essigsäurehaltigem Alkohol von $60 \%$. Von den erhaltenen Tinkturen wurde der Alkohol abdestilliert, das zurückbleibende Extrakt mit Wasser verdünnt und zur Trennung von fettem Oel und ausgeschiedenem Harz durch ein genässtes Filter filtriert. Dieses Filtrat wurde mit Bleiessig gefällt, abermals filtriert, ohne vorheriges Entbleien mit Natronlauge alkalisch gemacht und mit Chloroform ausgeschüttelt. Nach dem Abdestillieren des Chloroforms hinterblieb das Rohalkaloid als ein bräunliches Oel, welches keine Neigung zeigte, zu krystallisieren. Wir versuchten daher zunächst das Basengemisch in die einzelnen Bestandteile zu trennen und neutralisierten in dieser Absicht die Alkaloide mit Bromwasserstoffsäure. Indessen wollte es uns nicht gelingen, obgleich die einzelnen Krystallfraktionen verschiedenes Aussehen und verschiedenen Bromgehalt besassen, einheitliche Hydrobromide zu erhalten. Ein Vorversuch hatte ergeben, dass die Platindoppelverbindungen der Anagyrisalkaloide in ihren Löslichkeitsverhältnissen soweit von einander abwichen, dass man hoffen konnte, mit Hilfe dieser Verbindungen die reinen Alkaloide isolieren zu können. Deshalb verwandelten wir das Hydrobromid in Hydro- 
chlorid und versetzten die mit Salzsäure angesänerte Lösung desselben mit Platinchlorid. Sofort fiel ein mikrokrystallinischer Niederschlag von orangeroter Farbe aus. Derselbe wurde abgesaugt, mit wenig salzsäurehaltigem Wasser ausgewaschen und aus salzsäurehaltigem Wasser umkrystallisiert. Durch sorgfältig fortgesetzte fraktionierte Krystallisation dieses Niederschlages und des in der Mutterlauge enthaltenen Platinsalzes gelang es nun allerdings $\mathrm{zwei}$ offenbar verschiedene Platindoppelsalze $\mathrm{zu}$ isolieren. Die weitere Untersuchung lehrte indessen, dass man auf einfacherem Wege zu einer exakten Trennung der beiden Basen gelangen kann. Cytisin nämlich liefert zwar als freie Base mit Quecksilberchlorid einen weissen Niederschlag, die salzsaure Lösung dieses Alkaloides aber wird durch Sublimat nicht gefällt. Andererseits liefert das Anagyrincblorbydrat auch in saurer Lösung mit Quecksilberchlorid einen weissen, krystallinischen Niederschlag, dessen sich Hardy und Gallo is bereits für die Isolierung des Anagyrins bedient hatten. Wir führten daher die Trennung der beiden Basen schliesslich in der Weise aus, dass wir die mit Salzsäure angesäuerte Lösung der Rohbasen mit Quecksilberchlorid versetzten. Das entstehende Quecksilberdoppelsalz wirde mit sublimathaltigem Wasser ausgewaschen, und der Niederschlag sodann mit Schwefelwasserstofi zerlegt." "... Das Filtrat rom Anagyrinquecksilberchlorid wurde mit Schwefelwasserstoff entquecksilbert, die von letaterem befreite Lösung mit Kalilauge alkalisch gemacht, und das Cytisin mit Chloroform ausgeschüttelt. Beim Abdestillieren des Lösungsmittels hinterblieb das Cytisin als beim Erkalten direkt krystallisierende Masse zurück. “

Ich habe mich bei der Verarbeitung des grössten Teiles der mir zur Verfügung gewesenen Anagyrissamen genau dem oben skizzierten Verfahren angeschlossen. Es schien mir jedoch wünschenswert festzustellen, ob die Entbleiung der mit Bleiessig behandelten Rohalkaloidlösung yor dem alkalisch machen nicht zu einem weniger gefärbten und durch Nebenstoffe weniger verunreinigten Produkte führt. Ein in dieser Richtung angestellter Versuch lehrte, dass die Vornahme der Entbleiung in der That nur eine solch geringe Aufhellung zur Folge hat, dass der erzielte Effekt mit dem Verluste an Zeit und den in dem Bleisulfidniederschlage zurückgehaltenen Basen in keinem günstigen Verhältnisse steht.

Was Partheil's und Spasski's oben zitierte Angaben über die Versuche hinsichlich der Trennung des Cytisins vom Anagyrin anbetrifft, so kann ich dieselben im allgemeinen durch eigene Befunde bestätigen. Indessen wollte die völlige, exakte Trennung der beiden Basen, welche nach den beiden Autoren durch die Quecksilberchloridmethode ermöglicht sein soll, mir nicht gelingen. In allen Fällen erhielt ich zwar völlig anagyrinfreies Cytisin, doch stets mit mehr oder weniger Cytisin verunreinigtes Anagyrin, so dass ich gezwungen war, das Quecksilberdoppelsalz des letzteren in heissem Wasser von neuem zu lösen, das Quecksilber mittelst Schwefelwasserstoff zu entfernen 
und die von letzterem befreite Lösung des salzsauren Anagyrins abermals mit Quecksilberchlorid zu fällen. Abgesehen von der so hinzukommenden Umständlichkeit konnte mich dieses Ergebnis noch deshalb nicht zufriedenstellen, weil von den Quecksilbersulfidniederschlägen trotz der weitgehendsten und erschöpfendsten Behandlungsweise nicht unbeträchtliche Mengen der Basen hartnäckig zurückgehalten werden.

Aus diesen Gründen sah ich mich veranlasst, mein Augenmerk auf das Aufinden einer neuen ausgiebigeren und bequemeren Trennungsmethode zu richten.

Wie bereits E. Schmidt ${ }^{1}$ ) konstatierte und sich auch aus meinen Untersuchungen ergiebt, geht Cytisin, in konzentrierter alkoholischer Lösung mit Phenylsenföl in Reaktion versetzt, in kurzer Zeit quantitativ in Cytisinphenylthioharnstoff über, während Anagyrin, als tertiäre Base, unangegriffen bleibt. Die Schwerlöslichkeit dieses Harnstoffes in Alkohol und seine Unlöslichkeit in Wasser schienen mir für eine exakte Trennung der beiden Basen verwertbar zu sein, und stellte ich mehrere diesbezügliche Versuche an.

Für das Erste verfuhr ich derart, dass ich die Rohalkaloide in etwa der gleichen Menge absoluten Alkohols löste und diese Lösung mit überschüssigem Phenylsenföl versetzte. Die Mischung wurde, vor zu schneller Verdunstung des Lösungsmittels geschützt, drei Tage bei Seite gestellt und schliesslich, nachdem die Gegenwart von noch überschüssigem Phenylsenföl dargethan war, der abgeschiedene Cytisinthioharnstoff gesammelt. Die abgesaugte Flüssigkeit dunstete ich zur Vertreibung des überschüssigen Phenylsenföles bei mässiger Wärme bis zur Sirupdicke ein und nahm den Rückstand mit salzsäurehaltigem Wasser auf. Hierbei fand eine ziemlich betrăchtliche Abscheidung schmieriger Massen statt, welche jedenfalls zum Teil von Nebenprodukten der Einwirkung des Phenylsenföles auf den vorhandenen Alkohol, zum Teil aber, worauf die unerwartet mässige Ausbeute an Anagyrin hinwies, von verharzter Base herrühren dürften. Diese salzsaure Lösung lieferte, nachdem sie alkalisch gemacht, mit Chloroform ausgeschüttelt und von dieser Lösung das Chloroform abdestilliert worden war, die freie Base in Form einer amorphen, wenig gelblich gefärbten Masse, welche keine Neigung zur Krystallisation zeigte. Ein Teil der solcher Art gewonnenen Base wurde in das Platindoppelsalz übergeführt. Alle Krystallisationen einschliesslich der der letzten Mutterlaugen ergaben nur die typischen Krystalle des Anagyrinplatinchlorids, und konnten solche der beiden Cytisinplatinsalze nicht aufgefunden werden. Ich glaube mich daher zu der Annahme berechtigt, dass in dem angegebenen

1) Privat-Mitteilung.

Arch. d. Pharm. OexxxviII, Bds. 3. Heft. 
Wege bei richtiger Befolgung eine neue Methode der exakten Trennung der beiden Anagyrisalkaloide gefunden ist. Indessen sind mit der Anwendung dieses Verfahrens auch bemerkenswerte Nachteile verknüpft:

Erstens liegt in dem Cytisinthioharnstoff, wie meine späteren Versuche beweisen, ein relativ beständiger Körper vor, so dass die Zurückgewinnung des Cytisins nur auf umständliche Weise und auch dann nur schwierig möglich ist. Zweitens treten infolge sekundärer Vorgänge bei der Befreiung des Anagyrins von dem überschüssigen Phenylsenföle nicht unbedeutende Verluste an dieser Base durch deren tiefergehende Veränderung ein.

Was den ersten Punkt betrifft, so liess sich das oben vorgezeichnete Verfahren allerdings unter Benutzung der Quecksilberchloridmethode in solcher Weise abändern, dass die bequeme Rückgewinnung des Cytisins gesichert war, jedoch konnte den bei der Befreiung des Anagyrins vom überschüssigen Phenylsenföle eintretenden Verlusten an dieser wertvollen Base nicht gesteuert werden. Dieses abgeänderte Verfahren besteht darin, dass die beiden Basen zuerst mittelst der Quecksilberchloridmethode getrennt werden. Hierauf werden die von. mit niedergerissenen Verunreinigungen mehr oder weniger gelblich, oft bräunlich gefärbten Fällungen des Anagyrinquecksilberchlorids nach sorgfältigem Auswaschen mit sublimat- und salzsäurehaltigem Wasser mittelst Schwefelwasserstoff zerlegt und dem von letzterem durch Erwärmen befreiten Filtrate, nachdem es alkalisch gemacht ist, das cytisinhaltige Rohanagyrin mittelst Chloroform entzogen. Der nach dem Abdestillieren des Chloroforms verbleibende Rückstand wird in der gleichen Menge Alkohols gelöst und in einem verschliessbaren Gefässe mit Phenylsenföl versetzt. Nach dreitägigem Stehen an kühlem Orte werden die ansgeschiedenen Krystalle des Cytisintbioharnstoffes von der Flüssigkeit gesondert. Zur Gewinnung des reinen Aikaloids verfährt man nun, wie oben bereits beschrieben ist.

Trotz der exfolgten exakten Trennung der beiden Basen kann ich in Berücksichtigung der Kostspieligkeit des Ausgangsmateriales die von mir skizzierte Methode nur dort mit Vorbehalt empfehlen, wo die angeführten Nachteile unberücksichtigt bleiben können, oder der Zweck es erfordert. Ich selbst habe in der Folge wieder das von Partheil und Spasski angegebene Trennungsverfahren aufgenommen, dasselbe jedoch in einem Punkte umgeändert und hierdurch ein Anagyrin gewonnen, welches nur noch so geringe Mengen von Cytisin enthielt, dass dieselben bei dem spezielleren Studium des Anagyrins nicht mehr störend wirkten. Ich löste nämlich das Rohalkaloidgemisch in absolutem Alkohol auf und erzielte durch Hindurchleiten von trockenem 
Salzsäuregas aus der gefärbten Lösung eine nahezu weisse Fällung aus den Chloriden der beiden Basen bestehend.

Dieses Salzgemisch wurde nach sorgfältigem Abpressen nach Massgabe der Quecksilberchloridmethode weiter behandelt. Es hat den Anschein, als ob die den Rohbasen noch anhaftenden Verunreinigungen bei der Trennung mittelst Quecksilberchlorid einen störenden Einfluss ausübten.

Da bei der Prüfung einer mit Salzsäure angesäuerten Probe der wiederholt mit Chloroform ausgeschüttelten, alkalischen, wässerigen Rohalkaloidlösung mit Goldchlorid noch eine beachtenswerte Fällung eintrat, sah ich mich veranlasst, dem Studium der Frage, ob in den Anagyrissamen neben Cytisin und Anagyrin ein oder mehrere andere Pflanzenbasen vorkommen, näher zu treten. $\mathrm{Zu}$ diesem $Z$ wecke neutralisierte ich die bezeichnete Flüssigkeit mit Salzsäure und dampfte sie auf dem Wasserbade bis auf ein kleines Volumen ein. Durch Zumischen einer hinreichenden Menge von Seesand und Soda und nunmehr erfolgendes weiteres Erhitzen auf dem Dampfbade resultierte schliesslich eine feste bröckliche Masse, welche in einem geräumigen Perkolator der wiederholten Extraktion mit Chloroform und endlich mit starkem Alkohol unterworfen wurde. Von diesen Auszügen wurden Chloroform und Alkohol abdestilliert, und der hinterbleibende Rückstand in wässeriger Liösung zuvörderst mit Bleiessig gereinigt. Die abfiltrierte und entbleite Lösung wurde, nachdem sie alkalisch gemacht war, mit Chloroform erschöpft und nach dem Abdestillieren des letzteren ein geringer, firnisartiger Rückstand gewonnen. Bei der Prüfung des letzteren mittelst Platinchlorid konnte nur die Anwesenheit von wenig Anagyrin und etwas mehr Cytisin dargethan werden.

Diesem Befunde gemäss, ist mit hoher Wahrscheinlichkeit anzunehmen, dass sich in den Anagyrissamen neben Cytisin und Anagyrin keine weiteren Alkaloide, wenigstens nicht in greifbarer Menge, vorfinden.

Schliesslich wäre noch in betreff des Mengenverhältnisses, in dem die beiden Basen in den Anagyrissamen vorkommen, zu bemerken, dass dieses durchaus kein konstantes ist. Bei einem Posten Anagyrissamen, von der Firma Merck-Darmstadt bezogen, stellte sich das Mengenverhältnis zwischen Cytisin und Anagyrin annähernd wie 3:1. Aus einer später bezogenen Quantität Samen, welche von der genannten Firma nen beschaffit werden musste, wurde etwas mehr Anagyrin gewonnen, so dass sich obiges Verhältnis wie $3: 2$ gestaltete.

Als Ausgangsmaterial für die an dieser Stelle niedergelegten speziellen Untersuchungen über das Anagyrin diente ausschliesslich das bromwasserstoffsaure Salz desselben, welches aus Wasser in sehr 
charakteristischen Tafeln krystallisiert, während für die Butylierungsversuche des Cytisins durchgehend ein aus den Samen von Cytisus laburnum gewonnenes Cytisin zur Verwendung gelangte, bei dem also eine Beimischung von Anagyrin ausgeschlossen war.

\section{Anagyringoldchlorid: $\mathrm{C}^{15} \mathrm{H}^{22} \mathrm{~N}^{2} \mathrm{O} \cdot \mathrm{HAu} \mathrm{Cl}^{4}$.}

Das Golddoppelsalz des Anagyrins, welches durch Fällung der salzsauren Lösung der freien Base mittelst Goldchlorid als zitronengelber Niederschlag erhalten wird, diente in erster Linie zur Ermittelung der molekularen Zusammensetzung des Anagyrins. Es ist, da es nicht so leicht in gut krystallisiertem Zustande gewonnen werden kann, bisher meist nur in amorpher oder in krystallinischer Form analysiert worden. Ich erhielt dieses Salz in Gestalt sehr dünnfilziger, hellgelber Nadeln, als ich zu einer heissen stark verdünnten Lösung des salzsauren Anagyrins Goldchlorid zufügte und die ungetrübte heisse Flüssigkeit langsam abkühlen liess. Der Schmelzpunkt dieser Krystalle liegt bei $209^{\circ}$, während er für das amorphe $\mathrm{Salz}$, in Uebereinstimmung mit Partheil's und Spasski's Angaben ${ }^{1}$ ) bei 207-208 ${ }^{\circ}$ gefunden wurde. Klostermann ${ }^{2}$ ) fand denselben bei 210-211 ${ }^{\circ}$.

1. $0,1020 \mathrm{~g}$ des bei $100^{\circ}$ getrockneten amorphen Salzes hinterliessen nach dem Glühen $0,0344 \mathrm{~g} \mathrm{Au}=33,72 \%$.

2. $0,1588 \mathrm{~g}$ des bei $100^{\circ}$ getrockneten krystallinischen Salzes hinterliessen $0,0532 \mathrm{~g} \mathrm{Au} 33,50 \%$.

3. $0,1878 \mathrm{~g}$ desselben Salzes lieferten $0,0632 \mathrm{~g} \mathrm{Au}=33,65 \%$.

4. $0,2354 \mathrm{~g}$ lieferten nach dem üblichen Verfahren $0,2320 \mathrm{~g} \mathrm{Ag} \mathrm{Cl}=$ $0,0573 \mathrm{~g} \mathrm{Cl}=24,34 \%$.

5. $0,2874 \mathrm{~g}$ des bei $100^{\circ}$ getrockneten Salzes lieferten $0,3248 \mathrm{~g} \mathrm{CO}=$ $0,08858 \mathrm{~g} \mathrm{C}=30,82 \%$ und $0,1060 \mathrm{~g} \mathrm{H}^{2} \mathrm{O}=0,01177 \mathrm{~g} \mathrm{H}=4,09 \%$.

6. $0,3222 \mathrm{~g}$, bei $100^{\circ}$ getrocknet, ergaben nach $\mathrm{D} \mathrm{umas} 12,5 \mathrm{ccm} \mathrm{N}$ (auf normalen Druck und Temperatur reduziert) $=0,0157025 \mathrm{~g} \mathrm{~N}=4,87 \%$.

$\begin{array}{rcccc}\text { Gefunden: } & \begin{array}{c}\text { Berechnet für } \\ \text { C15 } \mathrm{H}^{22} \mathrm{~N}^{9} \text { O.HAuCl4: }\end{array} & \begin{array}{c}\text { Hardy u. } \\ \text { Gallois: }\end{array} & \begin{array}{c}\text { Partheil } \\ \text { u. Spasski: }\end{array} & \begin{array}{c}\text { Kloster- } \\ \text { mann: }\end{array} \\ \% & \% & \% & \% & \% \\ \text { C. } 30,82 & 30,73 & 28,55 & - & 30,75 \\ \text { H. } 4,09 & 3,92 & 3,38 & - & 3,66 \\ \text { Cl. } 24,34 & 24,24 & 24,50 & 24,42 & - \\ \text { N. } 4,87 & 4,78 & 5,20 & - & - \\ \text { Au. } 33,72 & 33,57 & 33,79 & 33,93 & 33,76 \\ 33,50 & & & 33,80 & \\ 33,65 & & & 33,61 & \end{array}$

1) Apoth.-Ztg. 1895, No. 103.

2) Inaug.-Diss. Marburg 1898. 
Für die von Hardy und Gallois aufgestellte Formel, welche ebenso wie die von Klostermann und mir acceptierte das Molekulargewicht 246 besitzt, berechnen sich die Kohlenstoff- und Wasserstoffwerte eines Golddoppelsalzes wie folgt:

$$
\begin{gathered}
\mathrm{C}^{14} \mathrm{H}^{18} \mathrm{~N}^{2} \mathrm{O}^{2} \cdot \mathrm{H} \mathrm{AuCl}^{4} \\
\text { C. } \quad 28,68 \% \\
\text { H. } \quad 3,24 \%
\end{gathered}
$$

während die Werte für Chlor, Gold und Stickstoff naturgemäss dieselben sind, wie sie die Formel $\mathrm{C}^{15} \mathrm{H}^{22} \mathrm{~N}^{2} \mathrm{O} \cdot \mathrm{HAuCl}{ }^{4}$ verlangt.

\section{Anagyrinplatinchlorid: $\mathrm{C}^{15} \mathrm{H}^{22} \mathrm{~N}^{2} \mathrm{O} \cdot 2 \mathrm{HCl} \cdot \mathrm{PtCl}^{4}+1 \frac{11 / 2}{2} \mathrm{H}^{2} \mathrm{O}$.}

Dieses in kaltem und kochendem Wasser auch bei Gegenwart von Salzsäure schwer lösliche Doppelsalz wurde zu wiederholten Malen in der gebräuchlichen Weise dargestellt und entweder in schönen dunkelroten, aus verwachsenen Nadeln bestehenden Drusen, die oft die ansehnliche Grösse von $1 \frac{112}{2} \mathrm{~cm}$ Durchmesser erreichten, oder in Gestalt einzelner oder sternförmig gruppierter orangeroter, kurzer, gebogener Nadeln erhalten. Tafeln, wie sie Partheil und Spasski gewannen, hatte ich nicht Gelegenheit zu beobachten. Die von mir dargestellten Formen zersetzen sich bei dem Erhitzen, ohne zu schmelzen. Es scheint zwischen ihnen, wie aus der Zusammenstellung der Analysenresultate hervorgeht, ein kleiner Unterschied im Krystallwassergehalte obzuwalten. Und zwar enthalten die grossen Drusen anscheinend 2 Mol. Wasser, von denen beim Erhitzen auf $100-130^{\circ}$ bis zum konstanten Gewicht nur $1 \mathrm{Mol}$. oder wenig mehr ausgetrieben werden kann, während den einzeln ausgebildeten Nadeln wahrscheinlich ein Krystallwassergehalt von $1 \frac{1 / 2}{2} \mathrm{Mol}$. zukommt, welcher bei $100^{\circ}$ nicht in allen Fällen gänzlich abgegeben wird. Dies eigentümliche Verhalten gestattet es nicht, die Analysenbefunde des wasserfreien (d. h. bei $100^{\circ}$ und schliesslich bei $130^{\circ}$ bis zum konstanten Gewicht getrockneten) Doppelsalzes zur weiteren Bestätigung der für das Anagyrin angenommenen Formel heranzuziehen.

A. Einzelne Nadeln oder sternförmig gruppierte oder farrenblattartig verwachsene Krystalle:

H'0, 5-6 Stunden bei $1000: 2,84 ; 2,95 ; 2,25 \%$.

$\mathrm{H}^{2} \mathrm{O}, 12$ Stunden bei $130^{\circ}: 3,85 ; 4,02 ; 2,83 \%$.

Pt: 28,47; 28,58; 28,52; 28,46 (lufttrocken).

Berechnet für $\mathrm{C}^{15} \mathrm{H}^{22} \mathrm{~N}^{2} \mathrm{O} \cdot 2 \mathrm{H} \mathrm{Cl}, \mathrm{PtCl}^{4}+1 \frac{1}{2} \mathrm{H}^{2} \mathrm{O}: \mathrm{H}^{2} \mathrm{O} 3,95 ; \mathrm{Pt} 28,49$.

B. Drusenförmige Krystalle:

$\mathrm{H}^{2} \mathrm{O}, 5-6$ Stunden bei $100^{\circ}: 2,66 ; 2,98 ; 2,01$.

$\mathrm{H}^{2} \mathrm{O}, 12$ Stunden bei $130^{\circ}: 3,27 ; 2,98 ; 2,01$.

Pt: 28,17; 27,69; 27,96 (lufttrocken). 
Berechnet für $\mathrm{C}^{16} \mathrm{H}^{22} \mathrm{~N}^{2} \mathrm{O} \cdot 2 \mathrm{HCl}, \mathrm{PtCl}+2 \mathrm{H}^{2} \mathrm{O}$ : für $1 \mathrm{H}^{2} \mathrm{O} .2,60$; Pt. 28,12.

Hardy und Gallois: Pt. 29,55; C. 25,48; H. 3,59; N. 4,43; Cl. 31,03. Partheil: Ha 0. 4,05; Pt. 28,21.

Spasski: Pt. 28,44; 28,26; 28,46;28,56;28,47.

Klostermann: $\mathrm{H}^{3} \mathrm{O} .4,33$ (bei $130^{\circ}$ ); Pt. 28,26; 28,35; 28,17; 28,32; 28,57 (lufttrocken); C. 26,64; 26,54; H. 4,02;3,92 (lufttrocken).

Berechnet fïr $\mathrm{C}^{16} \mathrm{H}^{23} \mathrm{~N}^{2} \mathrm{O} \cdot 2 \mathrm{HCl}$, PtCl $+1 \frac{1}{2} \mathrm{H}^{2} \mathrm{O}:$ C. 26,$37 ; \mathrm{H} .3,95$.

Anagyrinquecksilberchlorid: $\mathrm{C}^{15} \mathrm{H}^{22} \mathrm{~N}^{2} \mathrm{O} \cdot \mathrm{HCl} \cdot \mathrm{HgCl}^{2}$.

Eine kleine Menge der bei der Trennung des Cytisins vom Anagyrin durch Quecksilberchlorid, bei Gegenwart vou Salzsäure, gewonnenen Fällung wurde zu wiederholten Malen aus sublimat- und salzsäurehaltigem Wasser umkrystallisiert, und bei langsamer Abkühlung und Verdunstung des Lösungsmittels grosse durchsichtige, nahezu farblose Sänlen erhalten, welche meist zu schönen grossen Gruppen angeordnet waren. Dieses Salz schmolz bei $225-226^{\circ}$. Die Analyse desselben lieferte folgende Werte:

I. $0,3690 \mathrm{~g}$ lufttrockene Substanz verloren bei $100^{0}$ getrocknet $0,0048 \mathrm{~g}$ $\mathrm{H}^{2} \mathrm{O}=1,30 \%$.

II. $0,4834 \mathrm{~g}$ derselben während einiger Wochen zwischen Fliesspapier getrockneten Krystalle verloren beim Erhitzen auf $100^{\circ} 0,0074 \mathrm{~g} \mathrm{H}^{2} \mathrm{O}=1,53 \%$.

III. $0,3642 \mathrm{~g}$ des bei $100^{\circ}$ getrockneten Salzes lieferten $0,1534 \mathrm{~g} \mathrm{Hg} \mathrm{S}$ $=-1322 \mathrm{~g} \mathrm{Hg}=36,29 \%$ und $0,2796 \mathrm{~g} \mathrm{Ag} \mathrm{Cl}=0,0691 \mathrm{~g} \mathrm{Cl}=18,97 \%$.

Gefunden:

\%

$\mathrm{H}^{2} \mathrm{O} . \quad 1,30 \quad 1,53$

Hg. 36,29

Cl. 18,97
Berechnet für $\mathrm{C}^{15} \mathrm{H}^{22} \mathrm{~N}^{2} \mathrm{O} \cdot \mathrm{HCl} \cdot \mathrm{HgCl}^{2}+1 / 8 \mathrm{H}^{2} \mathrm{O}$ :
$\%$

1,30

für $\mathrm{C}^{16} \mathrm{H}^{22} \mathrm{~N}^{2} \mathrm{O} \cdot \mathrm{HCl} \cdot \mathrm{Hg} \mathrm{Cl}^{3}$ :
19,24

Neben dieser wasserhaltigen Quecksilberdoppelverbindung gelangte ich auch zu einer wasserfreien, welche feine weisse, büschelförmige Nadeln bildete. Diese schmolzen unscharf bei $213-214^{\circ}$. Ihre chemische Zusammensetzung entspricht im übrigen der des oben beschriebenen Salzes.

$0,2602 \mathrm{~g}$ verloren bei $100^{\circ}$ getrocknet nichts an Gewicht und lieferten $0,1059 \mathrm{~g} \mathrm{H} \mathrm{gS}=0,0994 \mathrm{~g} \mathrm{Hg}=36,27 \%$, ferner $0,2012 \mathrm{~g} \mathrm{Ag} \mathrm{Cl}=0,0498 \mathrm{~g} \mathrm{Cl}$ $=19,13 \%$.

Gefunden:

Hg. 36,27\%

Cl. $19,13 \%$
Berechnet für $\mathrm{C}^{15} \mathrm{H}^{22} \mathrm{~N}^{2} \mathrm{O} \cdot \mathrm{HCl} \cdot \mathrm{HgCl}^{2}$ :

$36,13 \%$

19,24,

Ausser diesen Krystallformen wurde eine dritte beobachtet, nämlich weisse verwachsene Warzen, deren Schmelzpunkt bei $231^{\circ}$ lag. Aller 
Wahrscheinlichkeit nach ist dieses Salz mit dem kurz vorher beschriebenen identisch:

Es enthält kein Krystallwasser und stellt sich in seinem Aeusseren den bereits von Klostermann ${ }^{1}$ ) analysierten warzenförmigen Krystallen des Anagyrinquecksilberchlorids vom Schmp. 233-2340 an die Seite, für deren chemische Zusammensetzung Klostermann Werte ermittelte, welche sich mit denen von mir bei der Analyse obiger büschelförmiger Nadeln gefundenen im Einklang befinden: Hg. 36,0; Cl. 19,05.

Anagyrinhydrochlorid: $\mathrm{C}^{15} \mathrm{H}^{22} \mathrm{~N}^{2} \mathrm{O} \cdot \mathrm{HCl}+\mathrm{H}^{2} \mathrm{O}$.

Dieses Salz wird durch Neutralisation der freien Base mittelst Salzsäure und Verdunsten der Lösung im Exsiccator leicht erhalten. Zur Umkrystallisation ist besonders Methylalkohol sehr geeignet. Während es aus diesem Lösungsmittel in kleinen Tafeln zur Abscheidung gelangt, welche ein Molekül Krystallwasser enthalten, lassen sich auch wasserfreie Mikrokrystalle durch Fällung der abgekühlten Lösung des Chlorids in siedendem absolutem Alkohol mittelst Aether gewinnen.

Ebenso wie Klostermann beobachtete ich bei dem salzsauren sowohl, als auch bei dem bromwasserstoffsauren Anagyrin eigentümliche, grünliche oder bläuliche Färbungen, welche besonders lebhaft bei den durch langsame Verdunstung des Lösungsmittels erzielten, über $1 \mathrm{~cm}$ grossen Tafeln auftraten. Diese Färbungen kehrten auch bei wieder. holter Umkrystallisation stets wieder.

Auffallender Weise zeigte jedoch ein Teil der von mir dargestellten Salze eine schwach rosenrote Färbung. Dass diese Färbungen nicht durch ein besonders optisches Verhalten dieser Krystalle bedingt sind, konnte ich dadurch beweisen, dass es mir bei öfterer Behandlung der wässerigen Salzlösung mit frisch ausgeglühter Tierkoble -- allerdings unter reichlichem Materialverluste - gelang, vollkommen farblose Krystalle zu erzielen.

Das salzsaure Anagyrin löst sich sehr leicht in Wasser. Es schmilzt noch nicht bei $260^{\circ}$.

$0,2254 \mathrm{~g}$ verloren bei $100^{\circ}$ getrocknet $0,0138 \mathrm{~g} \mathrm{H}^{20}=6,12 \% ; 0,2116 \mathrm{~g}$ des getrockneten Salzes lieferten $0,1074 \mathrm{~g} \mathrm{AgCl}=0,02656 \mathrm{~g} \mathrm{Cl}=12,55 \%$. $0,2526 \mathrm{~g}$ verloren bei $100^{\circ}$ getrocknet $0,0142 \mathrm{~g} \mathrm{H} \mathrm{H}^{2} \mathrm{O}=5,95 \% ; 0,2384 \mathrm{~g}$ des getrockneten Salzes lieferten $0,5576 \mathrm{~g} \mathrm{CO} \mathrm{CO}^{2}=0,15207 \mathrm{~g} \mathrm{C}=63,78 \%$ und $0,1780 \mathrm{~g} \mathrm{H}^{2} \mathrm{O}=0,01977 \mathrm{~g} \mathrm{H}=8,29 \%$.

1) Inaug.-Diss, Marburg 1898. 


$$
\begin{array}{rrr}
\multicolumn{3}{c}{\text { Gefunden: }} \\
\mathrm{H}^{2} \mathrm{O} . & 6,12 & 5,95 \\
& & \\
\text { C. } & 63,78 & \\
\text { H. } & 8,29 & \\
\text { Cl. } & 12,55 &
\end{array}
$$

Berechnet für $\mathrm{C}^{15} \mathrm{H}^{22} \mathrm{~N} 20 \cdot \mathrm{HCl}+\mathrm{H}^{2} \mathrm{O}$ :

$$
5,99
$$$$
\text { für } \mathrm{C}^{15} \mathrm{H}^{22} \mathrm{~N}^{2} \mathrm{O} \cdot \mathrm{HCl} \text { : }
$$

63,71

8,14

12,56

Der Hardy - Gallo is 'schen Formel $\mathrm{C}^{14} \mathrm{H}^{18} \mathrm{~N}^{2} \mathrm{O}^{2} \mathrm{HCl}$ entsprechen folgende Werte: C. 59,46, H. 6,72\%.

Hardy und Gallois fanden für das entwässerte Salz: C. 59,68; H. 7,43 ; CI. 12,04 .

Klostermann ermittelte folgende Daten: $\mathrm{H}^{2} \mathrm{O} .5,94 ; \mathrm{HCl} .12,16$; 12,$0 ; 12,04 ; 12,07 ;$ C. 59,$81 ; 60,20 ; \mathrm{H} .8,01 ; 8,52$. Für $\mathrm{C}^{15} \mathrm{H}^{22} \mathrm{~N}^{2} \mathrm{O}$, $\mathrm{HCl}+\mathrm{H}^{2} \mathrm{O}$ berechnen sich: $\mathrm{HCl} .12,17$; C. 59,9 ; H. 8,31 .

Die krystallographische Untersuchung der Krystalle des chlorwasserstoffsauren Anagyrins, welche ich der Liebenswürdigkeit des Herrn Dr. Schwantke verdanke, ergab, dass dieselben mit denen des bromwasserstoffsauren Salzes isomorph sind.

Beobachtete Formen: c (001), b (010), m (110), d (101), f (021).

Die Ausbildung der Krystalle ist charakterisiert durch Vorherrschen von $d$ neben $m$ und c. Spaltbarkeit ziemlich vollkommen nach b (010). Optische Axenebene c (001).

Fur $p=1,814 ; d=1,00578 ; 1=2$ ermittelte Klostermann eine Ablenkung des polarisierten Lichtstrahles von $-5^{0} 12^{\prime}$ oder $\alpha_{[D]}=-142^{0} 28^{\prime}$.

Anagyrin-Hydrobromid: $\mathrm{C}^{15} \mathrm{H}^{22} \mathrm{~N}^{2} \mathrm{O} \cdot \mathrm{HBr}+\mathrm{H}^{2} \mathrm{O}$.

Um dieses charakteristische Salz darzustellen, wurde die freie Base mit Bromwasserstoffsäure neutralisiert und die Lösung zur Erzielung grosser tafelförmiger Krystalle der langsamen Verdunstung an licht- und staubgeschütztem Orte anheimgestellt. Das bromwasserstoffsaure Anagyrin schmilzt noch nicht bei $250^{\circ}$.

Herrn Dr. Schwantke's krystallographische Untersuchungen ergaben:

Krystallsystem: rhombisch-hemiëdrisch.

Beobachte Formen: e (001), m (110), p (111), o (112), d (101), f (021).

Die Krystalle sind tafelig nach c, meist dünntafelig, seitlich von p und m nicht selten auch o (schmal) begrenzt. Bei zunehmender Dicke der Krystalle tritt auch d, bisweilen noch $f$ hinzu.

Spaltbarbeit: ziemlich vollkommen nach (010). Ebene der optischen Axen (010), erste Mittellinie $b$, negativ.

$0,2268 \mathrm{~g}$ verloren bei $100^{\circ}$ getrocknet $0,0122 \mathrm{~g} \mathrm{H} \mathrm{H}^{2} \mathrm{O}=5,37 \% ; 0,2146 \mathrm{~g}$ des getrockneten Salzes lieferten $0,1236 \mathrm{~g} \mathrm{AgBr}=0,0525 \mathrm{~g} \mathrm{Br}=24,50 \%$. 
1. $0,2704 \mathrm{~g}$ verloren bei $100^{\circ}$ getrocknet $0,0142 \mathrm{~g} \mathrm{H}^{2} \mathrm{O}=6,25 \%$.

2. $0,2562 \mathrm{~g}$ des getrockneten Salzes lieferten $0,5160 \mathrm{~g} \mathrm{CO}=0,1407 \mathrm{~g}$ $\mathrm{C}=54,92 \%$ und $0,1576 \mathrm{~g} \mathrm{H}^{2} \mathrm{O}=0,01751 \mathrm{~g} \mathrm{H}=6,83 \%$.

\begin{tabular}{rccc}
\multicolumn{3}{c}{ Gefunden: } & Berechnet für $\mathrm{C}^{15} \mathrm{H}^{22} \mathrm{~N}^{2} \mathrm{O} \cdot \mathrm{HBr}+\mathrm{H}^{2} \mathrm{O}:$ \\
$\mathrm{H}^{2} \mathrm{O}$. & 5,37 & 5,25 & 5,21 \\
& & für $\mathrm{C}^{16} \mathrm{H}^{22} \mathrm{~N}^{20} \cdot \mathrm{HBr}:$ \\
C. & $\mathbf{5 4 , 9 2}$ & 55,04 \\
$\mathrm{H}$. & 6,83 & 7,03 \\
$\mathrm{Br}$. & $\mathbf{2 4 , 5 0}$ & 24,46
\end{tabular}

Für die Hardy - Gallo is' sche Formel $\mathrm{C}^{14} \mathrm{H}^{18} \mathrm{~N}^{2} \mathrm{O}^{2} \mathrm{HBr}$ berechnen sich C. 51,37, H. 5,81\%, Werte, welche sich mit den von mir ermittelten analytischen Daten nicht vereinbaren lassen, wohl stehen meine Werte aber im Einklang mit denen, welche Klostermann ermittelte.

Klostermann fand: $\mathrm{H}^{2}$ O. 5,$08 ; \mathrm{HBr} .23,73 ; 23,40 ;$ C. 51,90 ; 52,27 ; H. 6,83; 7,24; N 8,28; 8,60; 8,52. Für $\mathrm{C}^{16} \mathrm{H}^{22} \mathrm{~N}^{2} \mathrm{O}, \mathrm{HBr}+\mathrm{H}^{2} \mathrm{O}$ berechnen sich: $\mathrm{HBr} .23,47$; C. 52,17 ; H. 7,24 ; N. 8,11 .

\section{Anagyrin-Hydrojodid: $\mathrm{C}^{16} \mathrm{H}^{22} \mathrm{~N}^{2} \mathrm{O} \cdot \mathrm{HJ}+\mathrm{H}^{2} \mathrm{O}$.}

In der üblichen Weise dargestellt und aus starkem Alkohol umkrystallisiert bildet dieses Salz weingelbe, durchsichtige Nadeln, welche oft sternförmig gruppiert sind. Es schmilzt noch nicht bei $250^{\circ}$. Bei dem Aufbewahren über Schwefelsäure tritt allmählich Gelb- und endlich Braunfärbung ein.

$0,1980 \mathrm{~g}$ des Salzes verloren bei $100^{\circ}$ getrocknet $0,0094 \mathrm{~g} \mathrm{H} \mathrm{H}^{2} \mathrm{O}=4,74 \%$; $0,1886 \mathrm{~g}$ des getrockneten Salzes lieferten $0,1183 \mathrm{~g} \mathrm{AgJ}=0,0639 \mathrm{~J}=33,88 \%$.

\begin{tabular}{|c|c|}
\hline Gefunden: & Berechnet für $\mathrm{C}^{15} \mathrm{H}^{22} \mathrm{~N}^{2} \mathrm{O} \cdot \mathrm{HJ}$ \\
\hline $\mathrm{H}^{2} \mathrm{O} . \quad 4,74 \%$ & $\begin{array}{c}4,59 \% \\
\text { für } \mathrm{C}^{51} \mathrm{H}^{22} \mathrm{~N}^{2} \mathrm{O} \cdot \mathrm{H}\end{array}$ \\
\hline
\end{tabular}

J. $33,88 \%$ $33,95 \%$

Anagyrin-Perjodide.

Je nach den obwaltenden Bedingungen wurden verschieden zusammengesetzte Perjodide beobachtet. Benutzte ich zur Darstellung des jodwasserstoffsauren Salzes braungefärbte Jodwasserstoffsäure, so schieden sich alsbald schöne glänzende, braunrote Nadeln ab. Diesen kommt, wie die nachstehende Analyse zeigt, die Formel $\mathrm{C}^{15} \mathrm{H}^{22} \mathrm{~N}^{2} \mathrm{O}$. $\mathrm{HJ}+\mathrm{J}$ zu

$0,2582 \mathrm{~g}$ lieferten nach Carius $0,2405 \mathrm{~g} \mathrm{Ag} \mathrm{J}=0,1299 \mathrm{~g} \mathrm{~J}=50,30 \%$. Gefunden:

$\mathrm{J}_{2} \quad 50,30 \%$

Berechnet für $\mathrm{C}^{15} \mathrm{H}^{22} \mathrm{~N}^{20} \cdot \mathrm{HJ}+\mathrm{J}$ :

$50,69 \stackrel{q}{*}$ 
Ein zweites Perjodid erhielt ich durch Zufügen von jodkaliumhaltiger rauchender Jodwasserstoffsäure zu einer wässerigen Anagyrinlösung in Gestalt stahlgrauer, stark glänzender Nadeln oder Blättchen, welche ihre Unbeständigkeit durch Exhalation kräftigen Jodgeruches zu erkennen gaben. Dieser Verbindung ist vielleicht die Formel $\mathrm{C}^{15} \mathrm{H}^{22} \mathrm{~N}^{2} \mathrm{O} \cdot \mathrm{HJ}+2 \mathrm{~J}$ zuzuerteilen.

$0,1954 \mathrm{~g}$ dieses Perjodids lieferten nach Carius 0,2172 $\mathrm{g} \mathrm{AgJ}=0,1173 \mathrm{~g}$ $\mathrm{J}=60,03 \%$.
Gefunden:
$\mathrm{J}_{B} \quad 60,03 \%$
Berechnet für $\mathrm{C}^{16} \mathrm{H}^{22} \mathrm{~N}^{2} \mathrm{O} \cdot \mathrm{HJ}+2 \mathrm{~J}$ : $60,66 \%$

\section{Alkylderivate des Anagyrins.}

Wie bereits früher erwähnt wurde, hat Klostermann (l. c.) die Reaktionsfähigkeit des Anagyrins mit Methyljodid festgestellt und aus der Thatsache, dass das gebildete Alkylprodukt kein weiteres Molekül Jodmethyl addiert, das Anagyrin als eine Base von tertiärem Charakter erkannt. Ich habe diesen Versuch, für den eine weitere Bestätigung wünschenswert erschien, wiederholt und ausserdem auch das Verhalten des Anagyrins zu Aethyljodid einer Prüfung unterzogen. Während die Methylierung glatt verlief, zeigte es sich, dass die Aethylierung unter denselben Versuchsbedingungen nur unvollständig erfolgte.

\section{Anagyrin-Methyljodid: $\mathrm{C}^{15} \mathrm{H}^{22} \mathrm{~N}^{2} \mathrm{O} \cdot \mathrm{CH}^{8} \mathrm{~J}+\mathrm{H}^{2} \mathrm{O}$.}

In einer -gut schliessenden Druckflasche wurden 2,0 Anagyrin mit überschüssigem Jodmethyl und 2,0 Methylalkohol zusammengebracht und zor Beschleunigung der Reaktion im Wasserbade drei Stunden erhitzt. Nach dem Erkalten hatten sich einige gelblich-weisse Krystalle an den Gefüsswandungen angesetzt, die sich jedoch bei mehrtägigem Stehen nicht weiter vermehrten. Der Inhalt der Flasche wurde aus diesem Grunde durch Eindunsten von dem überschüssigen Methyljodid und Methylalkohol befreit und der Rückstand alsdann in heissem absolutem Alkohol gelöst. Da auch diese Lösung keine Neigung zur Krystallbildung zeigte, wurde sie in einer verschliessbaren Flasche mit einem gleichen Volumen Aether iuberschichtet und mehrere Tage hindurch sich selbst überlassen. Aut diese Weise resultierten blassgelblich gefärbte, feine, lange Nadeln, welche wahrscheinlich kein Krystallwasser enthielten, wenigstens war der Wasserverlust nach dem Trocknen des Krystallpulvers bei $100^{\circ}$ ein so geringer, dass er nicht einmal die Höhe des für 1/2 Mol. theoretisch berechneten Wassergehaltes erreichte. Die Ausführung einer Schmelzpunktbestimmung war, da das Anagyrin-Methyljodid, auf $220^{\circ}$ erhitzt, sich braun färbte, nicht möglich. 
$0,1918 \mathrm{~g}$ des bei $100^{\circ}$ getrockneten Salzes lieferten $0,1153 \mathrm{~g} \mathrm{AgJ}$ $=0,06231 \mathrm{~g} \mathrm{~J}=32,48 \%$.

Durch Umkrystallisation aus wenig siedendem Alkohol wurden sehr feine, durchsichtige, wasserhaltige Nadeln gewonnen.

$0,2736 \mathrm{~g}$ verloren bei $100^{\circ}$ getrocknet $0,0122 \mathrm{~g} \mathrm{H}^{2} \mathrm{O}=4,45 \%$.

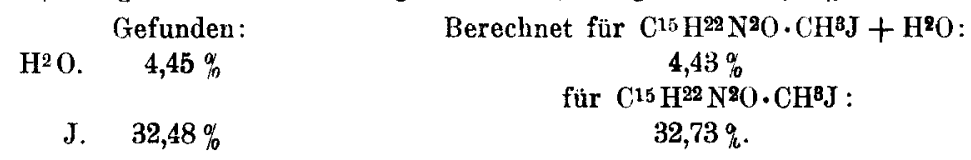

Klostermann erhielt das Anagyrin-Methyljodid aus Methylalkohol in wasserfreien Nadeln. Er fand: J. 32,69; 32,47; 32,55; 32,56 ; C. 46,70; H. 6,56. Für $\mathrm{C}^{15} \mathrm{H}^{22} \mathrm{~N}^{2} \mathrm{O} \cdot \mathrm{CH}^{3} \mathrm{~J}$ berechnen sich: J. 32,73 ; C. 49,$48 ;$ H. 6,44 .

Anagyrinmethylchlorid-Goldchlorid: $\mathrm{C}^{15} \mathrm{H}^{22} \mathrm{~N}^{2} \mathrm{O} \cdot \mathrm{CH}^{3} \mathrm{Cl} \cdot \mathrm{Au} \mathrm{Cl}^{3}$.

Zur Darstellung des Goldsalzes verfuhr ich derart, dass ich einen Teil des fast farblosen, krystallwasserhaltigen Anagyrinmethyljodids mit Chlorsilber umsetzte, das Filtrat mit Goldchloridlösung falllte, den Niederschlag sofort auf einem bereit gehaltenen Saugfilter absaugte und nur mit wenigen Tropfen salzsäurehaltigen Wassers nachwusch. Das zwischen Fliesspapier abgepresste Doppelsalz wurde sofort analysiert. Der Schmelzpunkt lag bei $198-199^{\circ}$.

$0,3144 \mathrm{~g}$ des bei $100^{\circ}$ getrockneten Salzes hinterliessen nach dem Glühen $0,1036 \mathrm{Au}=32,93 \%$. Gefunden:

Au. $\quad 32,93 \%$

Berechnet für $\mathrm{C}^{15} \mathrm{H}^{22} \mathrm{~N}^{2} \mathrm{O} \cdot \mathrm{CH}^{8} \mathrm{Cl} \mathrm{AuCl}^{3}$ : $32,78 \%$.

Anagyrinmethylchlorid-Platinchlorid: $\mathrm{C}^{15} \mathrm{H}^{22} \mathrm{~N}^{2} \mathrm{O} \cdot \mathrm{CH}^{3} \mathrm{Cl} \cdot \mathrm{HCl} \cdot \mathrm{PtCl}^{4}+\mathrm{H}^{2} \mathrm{O}$.

Zur Darstellung einer Platindoppelverbindung wurde ein Teil des krystallisierten Anagyrinmethyljodids mit Chlorsilber in das Chlorid verwandelt, und das Filtrat in üblicher Weise mit Platinchloridlösung gefällt. Das umkrystallisierte Salz stellte hellgelbrote, sehr kleine Nadeln dar, von denen infolge eintretender Schwarzfärbung keine Schmelzpunktbestimmung ausgeführt werden konnte.

$0,2008 \mathrm{~g}$ verloren bei $100-1250$ getrocknet $0,0050 \mathrm{~g} \mathrm{H}^{2} \mathrm{O}=2,49 \%$; $0,1958 \mathrm{~g}$ des getrockneten Salzes hinterliessen nach dem Glühen $0,0570 \mathrm{~g}$ $\mathrm{Pt}=29,11 \%$.

Gefunden: $\quad$ Berechnet für $\mathrm{C}^{15} \mathrm{H}^{22} \mathrm{~N}^{2} \mathrm{O} \cdot \mathrm{CH}^{8} \mathrm{Cl} \cdot \mathrm{HCl} \cdot \mathrm{PtCl}^{4}+\mathrm{H}^{2} \mathrm{O}$ :

$\mathrm{H}^{2} \mathrm{O} . \quad 2,49 \%$ $2,61 \%$

Pt. $\quad 29,11 \%$ für $\mathrm{C}^{16} \mathrm{H}^{22} \mathrm{~N}^{2} \mathrm{O} \cdot \mathrm{CH}^{8} \mathrm{Cl} \cdot \mathrm{HCl} \cdot \mathrm{PtCl}^{4}$ : $29,05 \%$. 
Klostermann fand $\mathrm{H}^{2} \mathrm{O} .2,37 ; 2,63 ;$ Pt. 28,59; 28,54 für wasserhaltiges, 28,87 für wasserfreies Salz.

Um zu entscheiden, ob das Anagyrin den Charakter einer sekundären oder tertiären Base trägt, hat Klostermann das Verhalten des Anagyrinmethyljodids bei erneuter Einwirkung von Jodmethyl studiert.

Klostermann fand hierbei, dass sich aus dem Anagyrin-Methyljodid das Jod nicht durch Kalilange, sondern entsprechend den Tetraalkylammoniumjodiden nur durch feuchtes Silberoxyd, unter Bildung einer Ammoniumbase, eliminieren lässt. Wurde die durch Silberoxyd erhaltene jodfreie Base von neuem mit Jodmethyl in Reaktion versetzt, so resultierte von neuem das als Ausgangsmaterial benutzte Anagyrinmethyljodid.

0,2247 $\mathrm{g}$ dieses Produktes lieferten 0,1369 $\mathrm{g}$ AgJ.

Gefunden:

J. $32,84 \%$
Berechnet für $\mathrm{C}^{15} \mathrm{H}^{22} \mathrm{~N}^{2} \mathrm{O} \cdot \mathrm{CH}^{3} \mathrm{~J}$ : $32,73 \%$.

Auch das aus diesem Jodid, nach Umsetzung mit Chlorsilber, dargestellte Platindoppelsalz entsprach in seinen Eigenschaften und in seiner Zusammensetzung dem oben beschriebenen Doppelsalze des Anagyrinmethylchlorids.

Gefunden: $H^{2} 0$. 2,96; Pt. 29,20; 29,33; C. 27,74; H. 4,50. Für $\mathrm{C}^{15} \mathrm{H}^{22} \mathrm{~N}^{2} \mathrm{O} \cdot \mathrm{CH}^{8} \mathrm{Cl}, \mathrm{HCl}+\mathrm{PtCl}^{4}$ berechnen sich: $\mathrm{Pt}$. 29,05; für die Verbindung $+\mathrm{H}^{2} \mathrm{O}:$ C. 27,$92 ; \mathrm{H} .4,07$.

Auch gegen salpetrige Säure verbält sich das Anagyrin, wie Klostermann konstatierte, indifferent. Aus dem Reaktionsprodukt konnte unverändertes Anagyrinhydrochlorid: $\mathrm{C}^{15} \mathrm{H}^{22} \mathrm{~N}^{2} \mathrm{O}$, $\mathrm{HCl}+\mathrm{H}^{2} \mathrm{O}$, und aus diesem Anagyrinplatinchlorid: $\mathrm{C}^{15} \mathrm{H}^{22} \mathrm{~N}^{2} \mathrm{O}$, $\mathrm{H}^{2} \mathrm{PtCl}^{6}+1 \frac{1}{2} \mathrm{H}^{2} \mathrm{O}$, dargestellt werden.

Dass das Anagyrin thatsächlich als tertiäre Base anzusehen ist, wird ferner durch den misslungenen Versuch, eine Verbindung mit Phenylsenföl darzustellen, bewiesen:

Reines amorphes Anagyrin wurde in wenig absolutem Alkohol gelöst, und nach Zusatz einer hinreichenden Menge Phenylsentöls die Mischung der Verdunstung überlassen. Es konnte nicht die geringste Krystallbildung beobachtet werden; schliesslich hinterblieb eine firnisartige Masse, der durch Behandlung mit Wasser das unveränderte Anagyrin entzogen werden konnte, während ein ganz geringer Riuckstand auf dem genässten Filter als Phenylsenföltröpfchen erkannt wurde. Eine Probe der wässrigen Liossung wurde mit Platinchlorid versetzt, und durch Umkrystallisation des Niederschlages die charakteristischen Nadeln des Anagyrinplatinchlorids gewonnen. 
Anagyrin-A ethyljodid: $\mathrm{C}^{15} \mathrm{H}^{22} \mathrm{~N}^{2} \mathrm{O} \cdot \mathrm{C}^{2} \mathrm{H}^{5} \mathrm{~J}+\mathrm{H}^{2} \mathrm{O}$.

Diesen Versuch stellte ich analog dem der Methylierung mit Jodäthyl und wenig absolutem Alkohol an. Indessen wurde bei diesem Verfahren nur ein kleiner Teil des angewandten Anagyrins im gewïnschten Sinne angegriffen. Den festen Anteil (I) des Reaktionsproduktes sonderte ich von dem flüssig gebliebenen und krystallisierte ihn aus heissem absolutem Alkohol um. Der flüssige Anteil wurde durch Eindunsten von überschüssigem Aethyljodid und Alkohol befreit, und der Rückstand schliesslich aus absolutem Alkohol unter Mitwirkung von Aether krystallisiert erhalten (II).

I. Umkrystallisierter, fester Anteil.

Weingelbe, zerbrechliche Nadeln, teils durchsichtig, teils undurchsichtig.

0,1930 der bei $100^{\circ}$ getrockneten Substanz lieferten $0,1165 \mathrm{~g} \mathrm{AgJ}=$ $0,062 y \mathrm{~g} \mathrm{~J}=32,59 \%$.

Gefunden:

J. $\quad 32,59 \%$
Berechnet für $\mathrm{C}^{15} \mathrm{H}^{22} \mathrm{~N}^{2} \mathrm{O} \cdot \mathrm{C}^{2} \mathrm{H}^{5} \mathrm{~J}$ : $31,59 \%$

für $\mathrm{C}^{15} \mathrm{H}^{22} \mathrm{~N}^{2} \mathrm{O} \cdot \mathrm{HJ}$ :

$33,95 \%$.

Da der von mir ermittelte Jodgehalt zwischen den für jodwasserstoffsaures Anagyrin und Anagyrin-Aethyljodid berechneten Werten liegt, so musste ich vermuten, dass die analysierten Krystalle ein Gemisch der genannten Verbindungen darstellten. Ich versuchte daher, durch Umkrystallisation bei langsamster Verdunstung und Auslesen gleich ausgebildeter Krystalle eine Trennung zu ermöglichen; dies ist mir denn auch nach vieler Mühe wenigstens annähernd gelungen.

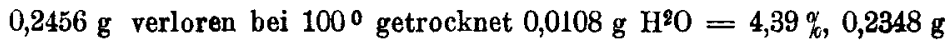
getrockneter Substanz lieferten 0,1387 g AgJ $=0,0750 \mathrm{~J}=31,94 \%$.

Gefunden:

$\mathrm{H}^{2} \mathrm{O} . \quad 4,39 \%$

J. $31,94 \%$
Berechnet für $\mathrm{C}^{15} \mathrm{H}^{22} \mathrm{~N}^{2} \mathrm{O} \cdot \mathrm{C}^{2} \mathrm{H}^{5} \mathrm{~J}+\mathrm{H}^{2} \mathrm{O}$ :

$4,28 \%$

für $\mathrm{C}^{15} \mathrm{H}^{22} \mathrm{~N}^{2} \mathrm{O} \cdot \mathrm{C}^{2} \mathrm{H}^{6} \mathrm{~J}$ :

$31,59 \%$.

II. Aus dem flüssigen Anteil.

Weingelbe, sehr zarte Nadeln.

$0,2100 \mathrm{~g}$ der bei $100^{\circ}$ getrockneten Krystalle lieferten 0,1316 g AgJ $=$ $0,07112 \mathrm{~g}=33,86 \%$. Gefunden:

J. $\quad 33,86 \%$

Berechnet für $\mathrm{C}^{15} \mathrm{H}^{22} \mathrm{~N}^{2} \mathrm{O} \cdot \mathrm{HJ}$ :

$33,95 \%$.

Demnach befand sich in dem flüssigen Anteil des obigen Reaktions. produktes nur jodwasserstoffsaures Anagyrin. 
Anagyrin-Aethylchlorid-Goldchlorid. $\mathrm{C}^{15} \mathrm{H}^{22} \mathrm{~N}^{2} \mathrm{O} \cdot \mathrm{C}^{2} \mathrm{H}^{5} \mathrm{Cl} \cdot \mathrm{AuCl}^{8}$.

Zur Darstellung dieses Salzes verwandte ich das Filtrat von der Jodbestimmung des Anagyrinäthyljodids. Der erhaltene amorphe Niederschlag wurde sofort abgesaugt, nur wenig mit salzsäurehaltigem Wasser ausgewaschen und analysiert.

$0,1986 \mathrm{~g}$ des bei $100^{\circ}$ getrockneten Salzes hinterliessen nach dem Glühen $0,0640 \mathrm{~g} \mathrm{Au}=32,22 \%$.

Gefunden:

Au $\quad 32,22 \%$

Berechnet für $\mathrm{C}^{15} \mathrm{H}^{22} \mathrm{~N}^{2} \mathrm{O} \cdot \mathrm{C}^{2} \mathrm{H}^{5} \mathrm{Cl} \cdot \mathrm{AuCl}^{3}$ : $32,04 \%$.

\section{Acetylierungsversuch.}

Die Oxydationsversuche, welche ich mit dem Anagyrin ausführte, (S. 208), um einen Einblick in die Konstitution des Anagyrinmoleküls zu gewinnen, veranlassten mich durch ihre Ergebnisse, das Studium über die Bindung des $\mathbf{0}$-atoms des Anagyrins in den Bereich der Untersuchungen zu ziehen. Für die Beurteilung der Analysenbefunde des erzielten Oxydationsproduktes schien mir vor allem die Priffung auf das Vorhandensein einer Hydroxylgruppe von Wichtigkeit zu sein.

Um über diesen letzterwähnten Punkt Aufschluss zu erhalten, kochte ich $2,0 \mathrm{~g}$ Anagyrin mit genügend Essigsäureanhydrid bei Gegenwart von wenig wasserfreiem essigsauren Natrium eine Stunde lang am Rückflusskühler. Die Flüssigkeit wurde hierauf unter zeitweiligem Zusatz von etwas starkem Alkohol bei gelinder Wärme eingedunstet, der Rückstand mit absolutem Alkohol erschöpft, und schliesslich die erzielte Lösung von dem essigsauren Natrium abfiltriert. Letztere diente nach der Verdünnung mit Wasser zur Darstellung einer Platindoppelverbindung in der üblichen Weise. Von den erzielten Krystallen, durch ihre Form und Farbe bereits als Anagyrinplatinchlorid gekennzeichnet, liess sich infolge eintretender Schwärzung und Zersetzung keine Schmelzpunktbestimmung ausführen.

$0,1942 \mathrm{~g}$ verloren bei $100^{\circ}$ und schliesslich bei $125^{\circ}$ getrocknet $0,0078 \mathrm{~g}$ $\mathrm{H}^{2} \mathrm{O}=4,02 \% ; 0,1864 \mathrm{~g}$ hinterliessen nach dem Glühen $0,0554 \mathrm{~g} \mathrm{Pt}=29,72 \%$.

Gefunden: Berechnet für $\mathrm{C}^{16} \mathrm{H}^{22} \mathrm{~N}^{2} \mathrm{O} \cdot 2 \mathrm{HCl} \cdot \mathrm{PtCl}^{4}+11 / 2 \mathrm{H}^{2} \mathrm{O}$ :

$\begin{array}{rc}\mathrm{H}^{2} \mathrm{O}=4,02 \% & 3,95 \% \\ \mathrm{Pt}=29,72 \% & \text { für } \mathrm{C}^{15} \mathrm{H}^{22} \mathrm{~N}^{2} 0.2 \mathrm{HCl} \cdot \mathrm{PtCl}^{4}:\end{array}$

Aus diesem Analysenbefunde erhellt, dass unter den angewandten Bedingungen keine Acetylierung des Anagyrins erfolgt ist. Es muss demnach als höchst wahrscheinlich angesehen werden, dass im Anagyrin keine Hydroxylgruppe enthalten ist, und stellt sich diese Base somit auch in dieser Eigenschaft dem Cytisin zur Seite. 


\section{Versuch des direkten Nachweises einer Butylgruppe im Anagyrin.}

J. Herzig und H. Meyer ${ }^{1}$ ) haben uns mit einer brauchbaren Methode bekannt gemacht, welche erlanbt, Methylgruppen am Stickstoff auf einfache und sichere Weise zum quantitativen Nachweis zu bringen. Ihre Methode beruht auf dem Verhalten der Hydrojodide solcher alkylhaltiger Basen bei höherer Temperatur, durch welche sie unter Bildung von Jodalkyl und der alkylfreien Base oder deren Zersetzungsprodukten tiefergreifende Veränderung erleiden. Aber nicht nur für den Nachweis von Methylgruppen, sondern auch für den von Aethylgruppen fand dieser Weg praktische Verwertung. Ob auch kohlenstoffreichere Alkylgruppen in analoger Weise zur exakten Bestimmung gebracht werden können, liess sich aus der mir zugänglichen Litteratur nicht feststellen. Um so mehr Interesse musste eine Untersuchung erwecken, welche auf das Verhalten des Anagyrinhydrojodids (als jodwasserstoffsaures Butyl-Cytisin aufgefasst) unter den Bedingungen dieser Methode gerichtet war.

Die Versuche wurden in dem von Herzig und Meyer angegebenen Apparate unter Beachtung der von ihnen geforderten Kautelen ausgeführt. Die Zersetzungstemperatur, - d. h. die Temperatur, bei welcher Trübung der vorgelegten alkoholischen Silbernitratlösung eintrat, - wurde zwischen $320-330^{\circ}$ gefunden.

I. $0,1612 \mathrm{~g}$ wasserfreies jodwasserstoffsaures Anagyrin lieferten $0,1298 \mathrm{~g}$ $\mathrm{Ag} \mathrm{J}=5,12 \stackrel{0}{\circ} \mathrm{CH}^{3}=19,52 \mathrm{C}^{4} \mathrm{H}^{9}$.

II. $0,2578 \mathrm{~g}$ derselben wasserfreien Substanz lieferten $0,0798 \mathrm{~g} \mathrm{Ag} \mathrm{J}=$ $1,94 \% \mathrm{CH}^{3}=7,48 \% \mathrm{C}^{4} \mathrm{H}^{9}$.

III. $0,2146 \mathrm{~g}$ lieferten ebenso $0,0726 \mathrm{~g} \mathrm{Ag} \mathrm{J}=2,15 \% \mathrm{CH}^{8}=8,20 \% \mathrm{C}^{4} \mathrm{H}^{9}$.

Für jodwasserstoffsaures Anagyrin berechnet sich $\mathrm{C}^{4} \mathrm{H}^{9}=15,24 \%$.

Wenn auch nach diesen Analysenbefunden der Beweis erbracht zu sein scheint, dass Anagyrin wenigstens eine $\mathrm{CH}^{3}$-Gruppe an Stickstoff enthält, (für jodwasserstoffsaures Anagyrin berechnet sich $\mathrm{CH}^{8}=4,01 \%$, so bin ich dennoch weit davon entfernt, diesen Schluss zu ziehen. Und zwar dies besonders deshalb, weil die bei sämtlichen Bestimmungen im Zersetzungskölbchen zurückgebliebene Substanz stets stark verkohlt war und mithin das Auftreten von Jodalkyl als sekundäres Zersetzungsprodukt nicht ausgeschlossen sein dürfte.

Nimmt man indessen an, dass Anagyrin thatsächlich ein Butylderivat des Cytisins ist, so lehrt obige Untersuchung, dass die Methode Herzig-Meyer nicht die quantitative Bestimmung kohlenstoffreicherer Alkyle am Stickstoff ermöglicht.

1) Monatshefte für Chemie, XV. 1894. 


\section{Oxydation des Anagyrins.}

Bei der Vorerwägung, in welcher Weise die Oxydation des Anagyring verlaufen würde, musste es als nicht ausgeschlossen erscheinen, dass, im Falle Anagyrin thatsächlich ein Butylabkömmling des Cytisins ist, eventuell die Seitenkette aboxydiert und ein Körper gebildet werde, der dem Cytisin oder einem Oxydationsprodukte desselben nahesteht. Diese Mutmassung hat sich nicht bestätigt, wie ich in der Folge näher ausführen werde.

Die Oxydation führte ich in der Weise aus, dass ich 10,0 Anagyrin in etwa 300,0 Wasser löste und nach und nach in der Kälte mit einer Baryumpermanganatlösung $(1: 10)$ versetzte, bis die bei jedesmaligem Zusatze des Permanganats entstehende und beim Umschwenken alsbald verschwindende Rotfärbung etwa fünf Minuten bestehen blieb. Der gebildete Manganniederschlag wurde abgesaugt und mehrere Male mit Wasser und Alkohol ausgekocht. Die vereinigten Filtrate wurden durch hinreichenden Zusatz verdünnter Schwefelsäure von Baryum befreit, auf dem Wasserbade eingeengt, alkalisch gemacht und endlich mehrmals mit Chloroform ausgeschüttelt. Nach dem Abdestillieren des letzteren hinterblieb ein blendend weisser Rückstand aus grossen strahligen Krystallen. Diese konnten durch Umkrystallisation aus Chloroform, Alkohol, Aceton oder Wasser nicht gereinigt werden, da sie in den genannten Lösungsmitteln ausserordentlich leicht löslich waren. Sie erwiesen sich hingegen als in Aether, Ligroin und Petroläther sehr schwer löslich. Durch Kombination von Chloroform mit Ligroin oder Petroläther gelang es mir, ein für das Anagyrinoxyd ${ }^{1}$ ) geeignetes Krystallisationsverfahren zu finden. lch verfuhr derart, dass ich das Anagyrinoxyd zunächst in wenig Chloroform löste und nunmehr etwa die vierfache Menge Ligroin oder Petroläther - ohne Rücksicht auf die eintretende starke Trübung - zufügte. Erwärmte ich diese Mischung auf dem Dampfbade, so klärte sie sich anfänglich auf. Alsbald aber setzten sich schöne, grosse seidenweiche Nadeln an den Gefässwandungen an, oder es bildeten sich bei dem Abkühlen sternförmige kleinere Nadeln. Der Schmelzpunkt der reinsten Krystalle lag bei $195^{\circ}$. Ich habe von diesem neuen Körper einige Analysen ausgeführt, doch sehe ich davon ab, den hierbei erhaltenen Resultaten schon jetzt beweisende Kraft beizulegen, weil einerseits das Analysenmaterial noch nicht völlig rein war, andererseits es nicht ganz sicher

1) So will ich vorlüufig das Oxydationsprodukt des Anagyrins benennen, ohne mit diesem Ausdruck irgend eine nähere Bestimmung des Verlaufes der Oxydation geben $z u$ wollen. Diese behalte ich mir für weitere Untersuchungen vor. 
ist, dass das den Krystallen anhaftende Ligroin bei dem Trocknen bei $110^{\circ}$ vollständig abgegeben wurde. Die Analyse des später zu beschreibenden Goldsalzes lieferte Ergebnisse, welche weit mehr Aufschluss über den Verlauf der Oxydation geben können.

Das Anagyrinoxyd besitzt die Fähigkeit, empfindliches blaues Lackmuspapier zu röten, dennoch verhält es sich im übrigen wie eine Base, indem es nicht nur ein gut charakterisiertes Goldsalz, sondern auch ein Platin- und Quecksilberdoppelsalz bildet. Auch wurde die Existenz eines krystallwasserhaltigen, leicht löslichen salzsauren Salzes nachgewiesen.

Das optische Verhalten des Anagyrinoxyds ist dasselbe wie das des Anagyrins; sowohl in neutraler wässeriger, als in salzsaurer Lösung konnte im Laurent'schen Halbschattenapparat deutliche Linksdrehung konstatiert werden. Es schien mir ferner eine Prüfung, ob das Anagyrinoxyd ebenso wie Cytisin und Anagyrin die v. d. Moer'sche Reaktion liefert, von Interesse zu sein. Zu diesem Zwecke wurde, ebenso, wie es bei der Prüfung auf optische Aktivität geschehen war, aus gut ausgebildeten Krystallen des Golddoppelsalzes durch Behandlung mit Schwefelwasserstoff, Vertreiben des letzteren, alkalisch Machen und schliessliche Isolirung des fraglichen Körpers mittelst Chloroform ein einwandfreies Material dargestellt. Die Reaktion trat mit einer Schärfe ein, die nichts zu wünschen übrig liess. Ich schliesse aus dieser Thatsache, dass sich bei dem Oxydationsprodukte der dem Cytisin und Anagyrin eigentümliche chemische Charakter erhalten hat.

\section{Anagyrinoxyd-Goldchlorid.}

Zur Darstellung des Goldsalzes verfährt man am vorteilhaftesten in der Weise, dass man zu einer salzsauren mit einem gleichen Volumen Alkohol versetzten und bis zum Sieden erhitzten Lösung des Anagyrinoxyds eine hinreichende Menge Goldchloridlösung, welche mit Alkohol versetzt und ebenfalls etwas erwärmt ist, hinzufügt. Nach dem Erkalten und längeren Stehen resultieren auf diese Weise lange, hellgelbe oder goldgelbe, durchsichtige Nadeln. Krystallisiert man diese aus heissem Alkohol bei langsamster Verdunstung um, so lassen sich kräftig ausgebildete, dunkel-goldgelbe durchsichtige Säulen gewinnen. Sehr häufig wurde auch eine farrenblattartige, sehr schöne Verwachsung der Krystalle beobachtet. Nach der krystallographischen Untersuchung, welche Herr Dr. Schwantke die Güte hatte auszuführen, stellt das Anagyrinoxydgoldchlorid kleine, bräunliche, stark glänzende prismatische Krystalle des rhombischen Systems dar, Prismenwinkel $=80^{\circ} 32^{\prime}$. Die spitze Kante ist durch die (sehr schmale) Längsfläche gerade abgestumpft. Am freien Ende die Basis. Doppel- 
brechung sehr stark, optische Axenebene wahrscheinlich die Basis. Der Axenwinkel ist sehr klein, so dass die Prismenflächen im kcnvergenten Lichte das Bild schief zur Axe getroffener Platten einaxiger Krystalle gewähren.

Eine zweite Krystallisation des Goldsalzes, haarförmige, gelbliche, seidenglänzende Nadeln, erwies sich durch die analogen optischen Eigeuschaften (sehr starke Doppelbrechung und sehr kleiner Axenwinkel) und den approximativ aut $81^{\circ}$ bestimmten Prismenwinkel als mit der vorigen identisch.

Das Goldsalz ist in kaltem und heissem Wasser, auch bei Gegenwart von Salzsäure nur schwer, unter geringer Reduktion, löslich. Der Schmelzpunkt liegt bei $225^{\circ}$.

Bei der Analyse wurden folgende Durchschnittswerte gefunden, auf deren Interpretation und Verwertung ich später weiter eingehen will.

$$
\begin{aligned}
\mathrm{Au} & =32,58 \% \\
\mathrm{Cl} & =23,96 \% \\
\mathrm{C} & =30,5 \% \\
\mathrm{H} & =3,8 \%
\end{aligned}
$$

Da dem Anagyringoldchlorid der höhere Goldgehait von 33,57\% entspricht, so muss bei der Oxydation des Anagyrins eine Verbindung mit höherem Molekulargewichte, durch Autnahme von Sauerstoff, in der einen oder anderen Weise gebildet worden sein.

\section{Anagyrinoxyd - Platinchlorid.}

Während das Anagyrin mit Platinchlorid ein in kaltem und heissem Wasser ausserordentlich schwer lösliches Doppelsalz liefert, ist das Anagyrinoxyd-Platinchlorid durch seine Leichtlöslichkeit gekennzeichnet. Es krystallisiert aus Wasser in sternförmig angeordneten Nadeln. Der Schmelzpunkt liegt bei $240^{\circ}$; gleichzeitig mit dem Schmelzen tritt Schwarzfärbung und Zersetzung ein.

$0,2348 \mathrm{~g}$ verloren bei $100^{\circ}$ getrocknet $0,0118 \mathrm{~g} \mathrm{H}^{2} \mathrm{O}=5,04 \%$.

$0,2230 \mathrm{~g}$ des getrockneten Salzes hinterliessen nach dem Giühen $0,0656 \mathrm{~g} \mathrm{Pt}=29,41 \%$.

Anagyrinoxyd-Quecksilberchlorid.

Dieses Salz ist ebenfalls für das Oxydationsprodukt höchst charakteristisch. Von dem Anagyrinquecksilberchlorid unterscheidet es sich dadurch, dass es in salzsäurehaltigem Wasser bedeutend leichter löslich ist. Seine Gewinnung macht nicht die geringste Schwierigkeit.

Nach Herrn Dr. Schwantke's Untersuchungen sind die äusserst leicht zerbrechlichen, stark glänzenden, farblosen Krystalle prismatisch, dünntafelig nach der einen Fläche, welche die spitze Kante eines 
Prismas von $66^{\circ}$ gerade abzustumpfen scheint (es konnte nur auf Schimmereinstellung gemessen werden). Die eine Kante der Kombination war noch durch eine weitere Fläche unter cirka $21^{\circ}$ zur Tafelfläche schief abgestumpft. Durch skelett-artiges Wachstum zeigen die Krystalle an den Seiten einspringende Winkel und einen u-förmigen Durchschnitt. Endflächen am freien Ende waren nicht zu beobachten. Auslöschung gerade, parallel der Längsrichtung. Auf der Tafelfäche sieht man im konvergenten Lichte eine Axe seitlich austreten. Krystallsystem monoklin?

Das Salz sehmilzt bei $226-227^{\circ}$. Es enthält kein Krystallw asser. $0,2896 \mathrm{~g}$ des bei $100^{\circ}$ getrockneten Salzes lieferten $0,0934 \mathrm{~g} \mathrm{HgS}=$ $35,06{ }_{\%}^{o} \mathrm{Hg}$ und $0,1731 \mathrm{~g} \mathrm{Ag} \mathrm{Cl}=0,0428 \mathrm{~g} \mathrm{Cl}=18,64 \%$.

\section{Untersuchungen zur Feststellung des Oxydationsverlaufes.}

Die ursprüngliche Meinung, dass eine im Anagyrin vielleicht vorhandene - $\mathrm{CH}^{2}$. $\mathrm{OH}$ Gruppe bei der Oxydation in - $\mathrm{COH}$ oder $\mathrm{COOH}$ übergegangen sein könnte, musste ich, nachdem der Nachweis einer OH-Gruppe im Anagyrin nicht gelungen war, als sehr unwahrscheinlich aufgeben. Eine andere Frwägung, der ich die Voraussetzung zu Grunde legte, dass das Anagyrin ein N-alkyliertes Cytisin ist, führte mich zu der Mutmassung, dass eine - $\mathrm{CH}^{3}$-gruppe der Seitenkette in $-\mathrm{CH}^{2} \cdot \mathrm{OH}$ umgewandelt sein könnte. Endlich veranlassten mich die Ergebnisse einer vor kürzerer Zeit von W. Wernick und R. Wolffenstein ${ }^{1}$ veröffentlichten Arbeit „Ueber die Einwirkung von Wasserstoffsuperoxyd auf $\mathrm{N}$-alkylierte Piperidinbasen" zu Uvtersuchungen in derselben Richtung.

Versuch, das Anagyrinoxyd zu acetylieren.

War die Oxydation derart verlaufen, dass aus einer - $\mathrm{CH}^{3}$.gruppe eine $-\mathrm{CH}^{2} \cdot \mathrm{OH}$-gruppe hervorgegangen war, so musste durch erfolgende Acetylierung der Nachweis des entstandenen Hydroxyls zu führen möglich sein. Um hierüber Aufschluss zu erhalten, kochte ich $0,2 \mathrm{~g}$ Anagyrinoxyd mit überschüssigem Essigsüureanhydrid und wenig wasserfreiem Natriumacetat eine Stunde am Rückflusskühler. Die Flüssigkeit wurde nunmehr vorsichtig zur Vertreibung des überschüssigen Essigsäureanhydrid eingedunstet, der Rückstand mit Alkohol aufgenommen und nach dem Ansäuren mit Salzsäure mittelst Goldchlorid in eine Doppelverbindung übergeführt. Der entstandene hellgelbe Niederschlag lieferte nach der Umkrystallisation aus Alkohol gelbe, durchsichtige Nadeln, welche bei $225^{\circ}$ schmolzen.

$0,2166 \mathrm{~g}$ lieferten nach dem Glühen $0,0702 \mathrm{~g}$ Au $=32,41 \%$.

1) Ber. XXXI, S. 1553 . 
Aus dem Resultate dieser Goldbestimmung in Verbindung mit dem der Schmelzpunktbestimmung geht hervor, dass unter obigen Bedingungen keine Acetylierung des Anagyrinoxyds erfolgt ist. Mithin musste die Annahme, dass bei der Oxydation eine Hydroxylgruppe enstanden sein könnte, sehr zweifelhaft erscheinen

\section{Versuch, das Anagyrinoxyd zu benzoylieren.}

Da genugsam Fälle bekannt sind, bei denen der Nachweis von Hydroxyl infolge besonderer Konstellation der übrigen Elemente nicht durch Acetylierung erbracht werden konnte, studierte ich, um das negative Resultat der vorgenommenen Acetylierung weiter zu bestätigen, das Verhalten des Anagyrinoxyds zu Benzoësäureanhydrid. Zu diesem Zwecke schmolz ich in einem verschlossenen Röhrchen eine kleine Menge des Oxydationsprodukts mit einer genügenden Menge Benzoësäureanhydrid zusammen und erhitzte während zwei Stunden im Wasserbade. Der Inhalt wurde alsdann mit salzsäurehaltigem Wasser ausgezogen, und aus dem Filtrate ein Goldsalz dargestellt. Die erzielten Krystalle schmolzen bei $224^{\circ}$ und konnten somit, wie durch eine Goldbestimmung weiter bestätigt wurde, mit dem Goldsalz des unve:änderten Anagyrinoxyds identifiziert werden.

$0,2422 \mathrm{~g}$ hinterliessen nach dem Glühen $0,0792 \mathrm{~g} \mathrm{Au}=32,70 \%$.

Nach den bei der Acetylierung und Benzoylierung gesammelten Erfahrungen ist das Vorhandensein einer Hydroxylgruppe im Anagyrinoxyd höchst unwahrscheinlich.

Verhalten gegen Hydroxylamin.

Diese Untersuchung sollte darüber Aufschluss geben, $o b$ das Anagyrinoxyd ein doppelt gebundenes $\mathrm{O}$-atom enthält oder nicht. Die Versuchsanordnung war derart, dass ich eine kleine Menge des neuen Körpers mit etwas mehr als der theoretisch berechneten Menge salzsauren Hydroxylamins und soviel Natriumkarbonat, als zur Abscheidung des freien Hydroxylamins nötig, in wässeriger Lösung zusammenbrachte und das Gemisch erst bei gewöhnlicher Temperatur, schliesslich bei Dampfbadwärme sich längere Zeit selbst überliess. Die Flüssigkeit wurde eingedunstet, mit Alkohol aufgenommen, und daraus eine Golddoppelverbindung hergestellt. Die erzielten goldgelben Krystalle schmolzen bei $225^{\circ}$, stellten also das Doppelsalz des unveränderten Anagyrinoxyds dar. Der Nachweis eines doppelt gebundenen O-atoms konnte mithin nicht erbracht werden. Indessen könnte auch in diesem Falle die Ketonfunktion, ähnlich wie es Partheil (S. später) für das Cytisin annimmt, und ich für das Anagyrin als sehr wahrscheinlich erachte, infolge besonderer Konfiguration der übrigen Elemente paralysiert sein. 
Reduktionsversuche.

Die diesbezüglichen Versuche unternahm ich, veranlasst durch die Ergebnisse der bereits erwähnten Arbeit von W. Wernick und R. Wolffenstein ${ }^{1}$ ). Merling ${ }^{2}$ ) erhielt durch Einwirkung von Wasserstoffsuperoxyd und N-Methylpiperidin eine Verbindung, die er Oxymethylpiperidin nannte und durch folgende Konstitutionsformel erläuterte:

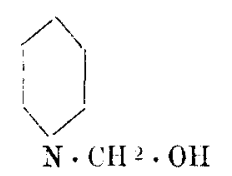

Durch Reduktion bildete sich aus dieser Base das Ausgangsmaterial zurïck. Während Wasserstoffsuperoxyd bei den sekundären Piperidinbasen auf die Bindung des $\mathrm{N}$-Atoms funktionsändernd ${ }^{\mathrm{B}}$ ) einwirkt, sollten sich also die N - alkylierten Piperidinbasen unter denselben Bedingungen wesentlich anders verhalten.

Wernick und Wolffenstein, welche dieses Verhalten für wenig wahrscheinlich ansahen, nahmen den von Merling beschriebenen Oxydationsversuch unter denselben Kautelen mit $\mathbf{N}$ - Aethylpiperidin vor, um das hierbei entstehende Reaktionsprodukt, - nach Merling'scher

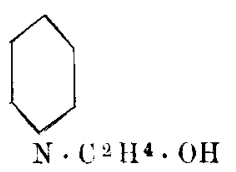
Auffassung also folgendermassen konstituiert - in seinen Eigenschaften mit eben derselben von Ladenburg ${ }^{4}$ ) beschriebenen, aus Piperidin mit Glykolchlorhydrin dargestellten Verbindung, dem Piperäthylalkamin, vergleichen zu können. Sie gelangten hierbei zu einer neuen Base, welche sich chemisch und physikalisch von der Ladenburg'schen unterschied und änderten auf Grund ihrer Untersuchungen, durch welche die lockere Bindungsweise des O-Atoms an Stickstoff bewiesen ist, die Merling'sche Bezeichnung Oxymethylpiperidin in Methylpiperidinoxyd und Oxyäthylpiperidin analog in Aethylpiperidinoxyd um, wie die folgenden Formeln illustrieren:
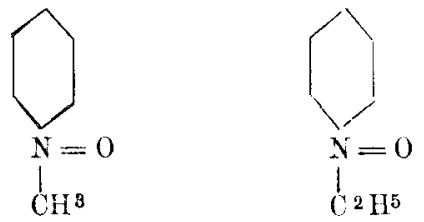

$\mathrm{Da}$ das Anagyrin ebenfalls als eine $\mathrm{N}$-alkylierte Base anzusehen ist, schien mir eine Untersuchung darüber angebracht zu sein, ob bei

1) Ber. XXXI, S. 1553.

2) Ber. XXV, S. 3123.

8) Ber. XXV, S. 2777. XXVI, S. 2991. XXVIII, S. 1459. XXIX, S. 1959. $\mathrm{XXX}, 2189$.

4) Ber. XIV, S. 1877 . 
dem Anagyrinoxyd ähnliche Verhältnisse bezüglich der Bindung des $\mathrm{O}$-Atoms vorlägen, wie sie die genannten Autcren für $\mathrm{N}$-alkylierte Piperidinbasen nachgewiesen haben. Wenn bei der Reduktion des Anagyrinoxyds Anagyrin zurückgebildet wurde, so war nicht nur über die Bindungsweise des $\mathrm{O}$-Atoms Klarheit geschaffen, sondern auch eine Inaktivierung des Anagyrins zu erwarten.

Das Anagyrinoxyd zeigte indessen bei der Einwirkung der verschiedenen Reduktionsmittel ein anderes, völlig stabiles Verhalten. Weder mit Zink und Salzsäure, noch mit schwefeliger Säure in der Kälte und der Wärme (in Druckflasche) konnte eine Sauerstoffabspaltung erzielt werden; stets wurde, wie durch Umwandlung der Reaktionsprodukte in die Goldsalze zu beweisen war, nur unverändertes Anagyrinoxyd zurückgewonnen. Da ferner letzteres nicht wie Aethylpiperidinoxyd aus Jodkaliumslösung Jod frei macht, scheint das $\mathrm{O}$-Atom nicht in peroxydartiger Bindung vorhanden zu sein.

Verhalten des Anagyrinoxyds zu salpetriger Säure.

Zum Nachweis, dass in dem Oxydationsprodnkt eine tertiäre Base vorliegt, wurde eine Probe desselben mit Natriumnitrit und Salz. säure auf dem Wasserbade erwärmt, die Lösung alkalisch gemacht und mit Chloroform ausgeschüttelt. Nach der Verdunstung des letzteren hinterblieb der ursprüngliche Körper, wie durch den Schmelzpunkt dieser Krystalle $-195^{\circ}-$, sowie durch den des daraus dargestellten Goldsalzes - $225^{\circ}$ - bewiesen wurde. Der tertiäre Charaktar des Anagyrinoxyds ist, da es keine Nitrosoverbindung liefert, demnach festgestellt.

Die Ergebnisse der vorstehenden Untersuchungen gestatten die endgültige Feststellung des chemischen Verhältnisses zwischen Anagyrinoxyd und Anagyrin noch nicht, da sie nur zu erkennen geben, wie der Oxydationsverlauf aller Wahrscheinlichkeit nach nicht war. Trotzdem scheint es mir zweckmässig, die von mir für das Anagyrinoxyd und dessen Derivate ermittelten Analysenwerte mit einem Abkömmling des Anagyrins in Vergleich zu stellen, in dem zwei H-Atome aboxydiert bezüglich durch ein $\mathrm{O}$-A tom vertreten sind.

Freies Anagyrinoxyd. Schmelzpunkt $195^{\circ}$.

Gefunden:

Mittelwert:

Berechnet für $\mathrm{C}^{15} \mathrm{H}^{20} \mathrm{~N}^{2} \mathrm{O}^{2}$ :

$\left.\begin{array}{rr}\text { C. } \quad 69,62 \\ \quad 68,73\end{array}\right\} \%$


Anagyrinoxyd-Goldchlorid. Schmelzpunkt $225^{\circ}$.
Gefunden:
Mittelwert:
Berechnet für $\mathrm{C}^{15} \mathrm{H}^{20} \mathrm{~N}^{2} \mathrm{O}^{2} \cdot \mathrm{HAuCl} 4$ :
C. $\left.\begin{array}{r}29,98 \\ 30,68\end{array}\right\} \%$
$30,33 \%$
$30,27 \%$
H. $\left.\begin{array}{r}3,56 \\ 4,05\end{array}\right\}$,
3,80
3,83 ,
Au. $\quad 32,41$
32,48
32,45
$\left.\begin{array}{l}32,64 \\ 32,71 \\ 32,56 \\ 32,82\end{array}\right\} "$
Cl. $23,96 \%$
32,58
32,79 ,
Anagyrinoxyd-Platinchlorid. Schmelzpunkt $240^{\circ}$.
Gefunden:
Berechnet für
$\mathrm{H}_{2} \mathrm{O} . \quad 5,04 \%$ $\mathrm{C}^{15} \mathrm{H}^{20} \mathrm{~N}^{2} \mathrm{O}^{2} \cdot 2 \mathrm{HCl} \cdot \mathrm{Pt} \mathrm{Cl}^{4}+2 \mathrm{H}^{2} \mathrm{O}$ :
Pt. $29,41 \%$
$5,08 \%$
für $\mathrm{C}^{15} \mathrm{H}^{20} \mathrm{~N}^{2} \mathrm{O}^{2} \cdot 2 \mathrm{HCl} \cdot \mathrm{PtCl}$ :
$29,05 \%$.

Anagyrinoxyd-Quecksilberchlorid. Schmelzpunkt 226-2270.

Gefunden:

Hg. $35,06 \%$

Cl. 18,64 n
Berechnet für $\mathrm{C}^{15} \mathrm{H}^{20} \mathrm{~N}^{2} \mathrm{O}^{2} \cdot \mathrm{HCl} \cdot \mathrm{Hg} \mathrm{Cl}^{2}$ :

$$
35,24 \%
$$

18,76 ,

\section{Versuche zur Partialsynthese des Anagyrins durch Butylierung des Cytisins.}

Einwirkung ron Isobutyljodid a f Cytisin.

Während Methyl- und Aethyljodid sich verhältnismässig leicht schon in der Kälte teilweise mit Cytisin vereinigen, wie die diesbezüglichen Versuche von Partheil ${ }^{1}$ ) gezeigt haben, findet bei dem Zusammenbringen von Cytisin und Isobutyljodid in der Kälte keine wahrnehmbare Wechselwirkung statt.

Zuerst wurde ein orientierender Versuch mit 1,5 g Cytisin vorgenommen, indem dieses mit überschüssigem Isobutyljodid in einer Druckflasche drei Stunden im Dampfbade erhitzt wurde. Der braungefärbte Inhalt der Flasche war nach dem Abkühlen teilweise fest geworden und liess innerhalb der am Boden haftenden zähen Masse deutlich weisse Partikelchen erkennen. Das darüber stehende überschüssige Isobutyljodid wurde abgegossen, und der Rückstand nach

1) Dieses Archiv 1892 . 
dem Lösen in wenig Wasser an lichtgeschütztem Orte über Schwefelsäure zur Krystallisation bei Seite gestellt. Als jedoch nach Verlauf einer Woche das Reaktionsprodukt nur zu einer harzartigen Masse eintrocknete, welche keine Neigung zur Krystallbildung zeigte, wurde sie in 96 prozent. Alkohol gelöst und wieder in der gleichen Weise der Verdunstung uberlassen; aber auch hierbei restierte nur eine zähe Masse. Diese wurde in Wasser gelöst, von ausgeschiedenem Harze durch Filtrieren befreit und mit Chlorsilber digeriert. Das Filtrat von dem überschüssigen Chlorsilber und gebildeten Jodsilber diente zur Darstellung eines Platindoppelsalzes. Die Versuche, ein Gold- und Quecksilberdoppelsalz zu erhalten, missglückten teils infolge eintretender Reduktion, teils infolge entstandenen Harzes. Das Platinsalz, welches schliesslich in Gestalt rötlich-gelber, warzenförmiger Krystalle erhalten wurde, schmolz unscharf bei $243^{\circ}$ unter vorheriger Braunfärbung.

$0,1958 \mathrm{~g}$ des bei $100^{\circ}$ getrockneten Salzes lieferten 0,0482 $\mathrm{g} \mathrm{Pt}=24,61 \stackrel{\alpha}{\circ}$.

Für die theoretisch möglichen Platinsalze eines butylierten Cytisins berechnen sich:

$$
\begin{aligned}
\text { (Mono-) } \mathrm{C}^{11} \mathrm{H}^{18}\left(\mathrm{C}^{6} \mathrm{H}^{9}\right) \mathrm{N}^{2} \mathrm{O} \cdot \mathrm{H}^{2} \mathrm{PtCl}^{6} & =29,67 \% \mathrm{Pt}, \\
\text { (Di-) }\left[\mathrm{C}^{11} \mathrm{H}^{13}\left(\mathrm{C}^{4} \mathrm{H}^{8}\right) \mathrm{N}^{2} \mathrm{O}^{8} \mathrm{H}^{2} \mathrm{PtCl} \mathrm{Cl}^{6}\right. & =21,57{ }_{n} \mathrm{Pt} .
\end{aligned}
$$

Der von mir ermittelte Platingehalt ist jedoch mit keinem dieser berechneten Werte in Einklang zu bringen. Dagegen stimmt die gefundene Menge sehr gut auf das Dicytisinplatinchlorid $\left(\mathrm{C}^{11} \mathrm{H}^{14} \mathrm{~N}^{2} \mathrm{O}\right)^{2} \mathrm{H}^{2} \mathrm{PtCl}^{6}$ - welches $24,63 \%$ Pt enthält. Auch das Aeussere der analysierten Krystalle passt gut auf das, was Partheil (1. c.) über Dicytisinplatinchlorid angiebt.

Da nicht zu erwarten stand, dass eine Butylierung des Cytisins in irgend einem Falle quantitativ gelingen würde, obiger Versuch ferner nicht entscheiden liess, ob die gewünschte Reaktion auch nur teilweise eingetreten war, wurde er mit einer grösseren Menge Cytisin, etwa $10,0 \mathrm{~g}$, wiederholt. Diesmal wurde jedoch der nach dem Erkalten teilweise festgewordene Inhalt der Druckflasche, nachdem das überschüssige Isobutyljodid abdestilliert worden war, mit absolutem Alkohol aufgeweicht, und hierdurch die Abscheidung eines weisslichgelben Pulvers erzielt. Die schliesslich nach öfterem Umkrystallisieren aus Alkohol gewonnenen weingelben Prismen schmolzen noch nicht bei $250^{\circ}$.

1. $0,2774 \mathrm{~g}$ verloren bei $100^{\circ}$ getrocknet $0,0146 \mathrm{~g} \mathrm{H}^{2} \mathrm{O}=5,26 \% ; 0,2628 \mathrm{~g}$ des getrockneten Salzes lieferten $0,1930 \mathrm{~g} \mathrm{Ag} J=39,68 \% \mathrm{~J}$.

Gefunden:

$\mathrm{H}^{2} \mathrm{O} . \quad 5,26 \%$

J. $39,68 \%$
Berechnet für jodw. Cytisin $\mathrm{C}^{11} \mathrm{H}^{14} \mathrm{~N}^{2} \mathrm{O} \cdot \mathrm{HJ}+\mathrm{H}^{2} \mathrm{O}$ :

$5,35 \%$

für C11 $\mathrm{N}^{14} \mathrm{~N}^{2} \mathrm{O} \cdot \mathrm{HJ}$ :

$39,93 \%$. 
Einem Butyl-Cytisinjodid würde ein Jodgehalt von $33,95 \%$ entsprechen.

Das Filtrat dieser Jodbestimmung wurde durch Salzsäure von dem überschüssigen Silbernitrat befreit und zur Darstellung eines Platindoppelsalzes in der üblichen Weise benutzt. Die erzielten hellgelben, durchsichtigen Krystalle zersetzten sich beim Erhitzen ohne zu schmelzen.

$0,2129 \mathrm{~g}$ verloren bei $100^{\circ}$ getrocknet $0,0142 \mathrm{~g} \mathrm{H}^{2} \mathrm{O}=6,66 \% ; 0,1957 \mathrm{~g}$ des getrockneten Salzes lieferten $0,0645 \mathrm{~g} \mathrm{Pt}=32,46 \%$.

Gefunden:

$\mathrm{H}^{2} \mathrm{O}, \quad 6,66 \%$

Pt. $32,46 \%$
Berechnet für Monocytisinplatinchlorid $\mathrm{C}^{11} \mathrm{H}^{14} \mathrm{~N}^{2} \mathrm{O} \cdot \mathrm{H}^{2} \mathrm{PtCl}^{6}+21 / 2 \mathrm{H}^{2} \mathrm{O}$ :

$6,98 \%$ für $\mathrm{C}^{11} \mathrm{H}^{14} \mathrm{~N}^{2} \mathrm{O} \cdot \mathrm{H}^{2} \mathrm{Pt} \mathrm{Cl}_{6}$ : $32,44 \%$.

Aus den Daten der Jod- und Platinbestimmung geht hervor, dass in den bei der Butylierung des Cytisins gewonnenen Krystallen nichts anderes als jodwasserstoffsaures Cytisin vorlag.

Die braune Flüssigkeit, von der das als jodwasserstoffsaures Cytisin erkannte Pulver durch Absaugen getrennt worden war, wurde zur weiteren Untersuchung mit Aether überschichtet. Es gelangten bei diesem Verfahren nach mehrtätigem Stehen jedoch nur vereinzelte, stark gefärbte Krystalle zur Abscheidung. Ich liess deshalb die Flüssigkeit bei mässiger Wärme eindunsten und löste den Rückstand nunmehr in jodwasserstoffsäurehaltigem Wasser auf. Es kam hierbei zur Abscheidung harzartiger Flocken, die abfiltriert wurden. Durch Eindunsten über Schwefelsäure restierte schliesslich ein gelblich braunes Liquidum, das nach langem Stehen geringe Krystallbildung zeigte. Jedoch blieb die Mühe, diese Krystalle von der firnisartigen Mutterlauge zu trennen, ohne Erfolg.

Schliesslich wurde mit einem Teile der in Wasser gelösten zähen Masse eine Umsetzung mit Chlorsilber vorgenommen, um durch das Studium herzustellender Gold- und Platinsalze die Frage entscheiden $\mathrm{zu}$ können, ob unter den angewandten Versuchsbedingungen überhaupt eine Butylierung erfolgt war. Die Versuche, ein Goldsalz darzustellen, schlugen fehl. In allen Fällen ballte sich das abgeschiedene amorphe Goldsalz bei dem Auflösen in salzsäurehaltigem Wasser und Alkohol zu harzigen, sehr schwer löslichen Tropfen zusammen, und die nach dem Erkalten der Lösungen abgeschiedene Substanz besass ganz dieselbe ölig-harzartige Beschaffenheit. Das Gold wurde deshalb aus der heissen Lösung der amorphen Substanz mit Schwefelwasserstoff ausgefällt, und nach dem Entfernen des letzteren zur Herstellung eines Platinsalzes geschritten. Nach vielem vergeblichen Bemühen gelang 
es mir schliesslich, gelblich-rote, warzenförmige Krystalle zu gewinnen, welche grosse Aehnlichkeit mit dem Dicytisinplatinchlorid besassen. $0,2542 \mathrm{~g}$ des bei $100^{\circ}$ getrockneten Salzes lieferten $0,0628 \mathrm{~g} \mathrm{Pt}=24,70 \%$. Gefunden:

Pt. $\quad 24,70 \%$ Berechnet für $\left(\mathrm{C}^{11} \mathrm{H}^{14} \mathrm{~N}^{2} 0\right)^{2} \mathrm{H}^{2} \mathrm{Pt} \mathrm{Cl}^{6}$. $24,61 \%$.

Der Rest des Rïckstandes wurde in Wasser gelöst, mit Kalilauge alkalisch gemacht und nunmehr mit Aether-Chloroform ausgeschüttelt. Die Lösung hinterliess nach dem Abdestillieren des Lösungsmittels einen zähen gelbbraunen Firnis, welcher mit salzsäurehaltigem Wasser aufgenommen, sodann mit Platinchloridlösung versetzt wurde. Hierbei kaun es nur zur Bildung gelatinöser Häutchen. Die Platindoppelsalzdarstellung verlief jedoch glatt, nachdem ich eine Reinigung mit obiger Flüssigkeit in der Art vorgenommen hatte, dass ich das Platin ausfällte. Aus dem schwefelwasserstofffreien Filtrate konnte ich nunmehr die für Monocytisinplatinchlorid charakteristischen glänzenden Nadeln mit Leichtigkeit erzielen.

Einwirkung von Isobutyljodid auf Cytisin bei Gegenwart von Isobutylalkohol.

Die Erfahrung lehrt, dass die Alkylierung mitunter durch die Gegenwart des korrespondierenden Alkoholes getördert wird. Versuchsweise wurden 5,0 Cytisin mit überschüssigem Isobutyljodid und 5,0 Isobutylalkohol in einer Druckflasche im Dampfbade während drei Stunden erhitzt. Der Inhalt war nach dem Abkühlen grösstenteils fest geworden und liess nach mehrtägigem Stehen deutlich krystallinisches Gefüge erkennen. Er wurde mit absolutem Alkohol aufgenommen, und das gesammelte gelblich-weisse Krystallgemenge aus $96 \%$ Alkohol umkrystallisiert. Die schliesslich gewonnenen weingelben Nadeln schmolzen noch nicht bei $250^{\circ}$.

$0,2952 \mathrm{~g}$ verloren bei $100^{\circ}$ getrocknet $0,0155 \mathrm{~g} \mathrm{H} \mathrm{H}^{2} \mathrm{O}=5,24 \% ; 0,2797 \mathrm{~g}$ des getrockneten Salzes lieferten 0,2069 $\mathrm{g} \mathrm{Ag} \mathrm{J}=0,1118 \mathrm{~g} \mathrm{~J}=39,97 \%$.

Gefunden:

$\mathrm{H}^{2} \mathrm{O}, \quad 5,24 ; 0$

J. $39,97 \%$
Berechnet für jodw. Cytisin

$\mathrm{C}^{11} \mathrm{H}^{14} \mathrm{~N}^{2} \mathrm{O} \cdot \mathrm{HJ}+\mathrm{H}^{2} \mathrm{O}$ :

für $\mathrm{C}^{11} \mathrm{H}^{14} \mathrm{~N}^{2} \mathrm{O} \cdot \mathrm{HJ}$ :

$39,93 \%$.

Die Flïssigkeit, von der das jodwasserstoffsaure Cytisin durch Absaugen getrennt worden war, diente nach der Umsetzung in das Chlorid zur Darstellung eines Platindoppelsalzes. Auch hier verhinderten anfänglich harzige Beimengungen die Krystallisation.

Nach wiederholter Reinigung dureh Ausfällen des Platins mit Schwefelwasserstoff und Ausschütteln des alkalisch gemachten Filtrates 
mit Aether-Chloroform gelang es schliesslich, eine krystallisierende Platinverbindung zu gewinnen. Leider reichte deren Menge zu einer quantitativen Bestimmung nicht aus. Das Salz besass ein von den beiden Cytisinplatinchloriden sehr verschiedenes Aeussere. Es stellte rote, undurchsichtige, gebogene Körnchen dar; sie wurden vorläufig auf bewahrt und später mit ähnlichen näher untersucht.

Einwirkung von Isobutyljodid auf Cytisin bei Gegenwart von Isobutylalkohol im zugeschmolzenen Rohre bei $150^{\circ}$.

In einem Cariusrohre wurden 5,0 Cytisin, 5,0 Isobutylalkohol und überschüssiges Isobutyljodid eingeschmolzen und während sechs Stunden auf $150^{\circ}$ erhitzt. Der Inhalt wurde nach dem Oeffnen des Rohres mit absolutem Alkohol versetzt, und alsdann eine Trennung des unveränderten Cytisins mit Phenylsenföl vorgenommen. $\mathrm{Zu}$ diesem Zwecke wurden Alkohol und überschüssiges Isobutyljodid durch $\mathrm{Ab}$ destillation entfernt und der bei mässiger Wärme erzielte Eindunstungsrückstand nach Zusatz von hinreichend Kalilauge mit Aether-Chloroform ausgeschüttelt. Nach dem Abdestillieren des Lösungsmittels hinterblieb eine braungelbe Masse, deren Lösung in absolutem Alkohol auf Zusatz von Phenylsenföl alsbald eine reichliche Abscheidung des leicht zu charakterisierenden Cytisinthioharnstoffes zeigte. Nach sechsstündigem Stehen wurde die Mutterlauge dieser Krystalle durch Eindampfen nach Zusatz von etwas Salzsäure von dem Ueberschusse an Phenylsenföl befreit. Ich versuchte nun, die wässerige Lösung des hierbei erhaltenen Rückstandes in ein Platinsalz zu verwandeln, indessen machten sich harzartige Anteile störend geltend. Schliessiich wurde eine sehr geringe Menge rötlicher Körner erhalten, welche eine gekrümmte Form aufwiesen und wegen ihrer Aehnlichkeit mit früher (s. oben) gewonnenen vereinigt wurden.

Einwirkung von Isobutylbromid auf Cytisin.

Dieser Versuch wurde mit 1,5 g Cytisin und einem Ueberschusse von Isobutylbromid in der üblichen Weise in einer Druckflasche ausgeführt. Die Reaktion suchte ich durch dreistündiges Erhitzen im Dampfbade zu bewerkstelligen. Der Inhalt hatte sich bei dieser Behandlung bräunlich gefärbt, liess aber nur geringe Krystallbildung an der Gefässwandung wahrnehmen. Nach mehrtägigem Stehen war jedoch die Flüssigkeit grösstenteils zu gelblich getärbten Krystallen erstarrt. Der darüber stehende flüssige Anteil hinterliess bei der freiwilligen Verdunstung einen kaum erwähnenswerten Rückstand.

Die gelblichen Krusten wurden mit absolutem Alkohol angeschlemmt und das nur noch wenig gefärbte Pulver nach dem Ab- 
saugen aus heissem Alkohol umkrystallisiert. Die erzielten grossen weissen Nadeln schmolzen noch nicht bei $250^{\circ}$.

0,2204 verloren bei $100^{\circ}$ getrocknet $0,0142 \mathrm{~g} \mathrm{H}^{2} \mathrm{O}=6,44 \% ; 0,2062 \mathrm{~g}$ des getrockneten Salzes lieferten $0,1435 \mathrm{~g} \mathrm{Ag} \mathrm{Br}=29,61 \% \mathrm{Br}$.

Gefunden :

$\mathrm{H}^{2} \mathrm{O} . \quad 6,44 \%$

Br. $29,61 \%$
Berechnet für bromw. Cytisin

$\mathrm{C}^{11} \mathrm{H}^{14} \mathrm{~N}^{2} \mathrm{O} \cdot \mathrm{HBr}+\mathrm{H}^{2} \mathrm{O}$ :

für $\mathrm{C}^{11} \mathrm{H}^{14} \mathrm{~N}^{2} \mathrm{O} \cdot \mathrm{HBr}$ :

$29,52 \%$.

In der Hauptsache war also nur bromwasserstoffsaures Cytisin gebildet worden. Doch lieferte auch hier die Mutterlauge der analysierten Krystalle nach der Ueberführung in das Chlorid neben den charakteristischen Krystallen des Monocytisinplatinchlorids geringe Mengen rötlicher, gekrümmter Körnchen.

Letztere wurden mit denen, welche bei den vorhergehenden Butylierungsversuchen resultierten, vereinigt und analysiert.

$0,1462 \mathrm{~g}$ verloren bei $100^{\circ}$ getrocknet $0,0062 \mathrm{~g} \mathrm{H}^{2} \mathrm{O}=4,24 \% ; 0,1400 \mathrm{~g}$ des getrockneten Salzes hinterliessen $0,0340 \mathrm{~g} \mathrm{Pt}=24,28_{0}^{\circ}$.

Diese rötlichen Körner konnten nicht als das Platinsalz eines Butyl-Cytisins angesehen werden; wahrscheinlich bestanden sie nur aus unreinem Dicytisinplatinchlorid, welches zwar gewöhnlich wasserfrei krystallisiert, aber bereits von Buchka und Magalbaes ${ }^{1}$ ) krystallwasserhaltig (2 Mol.) aufgefunden wurde.

\begin{tabular}{|c|c|}
\hline Gefunden: & $\begin{array}{l}\text { Berechnet für Dicytisinplatinchl } \\
\left(\mathrm{C}^{11} \mathrm{H}^{14} \mathrm{~N}^{2} \mathrm{O}\right)^{2} \mathrm{H}^{2} \mathrm{PtCl}^{6}+2 \mathrm{H}^{2}\end{array}$ \\
\hline $20 . \quad 4,24 \%$ & $\begin{array}{c}4,36_{\%}^{\%} \\
\text { für }\left(\mathrm{C}^{11} \mathrm{H}^{14} \mathrm{~N}^{2} \mathrm{O}\right)^{2} \mathrm{H}^{2} \mathrm{PtCl}^{6}:\end{array}$ \\
\hline Pt. $\quad 24,28 \%$ & $24,63 \%$. \\
\hline
\end{tabular}

Einwirkung von Isobutyljodid auf Cytisin in alkoholischer Lösung bei Gegenwart von Kaliumhydroxyd.

Dem in einer Druckflasche befindlichen Cytisin wurde eine gleiche Menge festen Kalihydrats, ferner überschüssiges Isobutyljodid urd wenig absoluter Alkohol zugefügt. Nach dreistündlichem Erhitzen im Wasserbade säuerte ich den erkalteten braunen, flüssigen Inhalt mit Jodwasserstoffsäure an und vertrieb den Alkohol sowie das überschüssige Alkyljodid durch vorsichtiges Eindunsten. Der zähe Rückstand wurde mit Wasser aufgenommen, mit Chlorsilber umgesetzt, die erzielte Lösung schliesslich, da aus einer Probe die Darstellung einer Platinverbindung nicht gelingen wollte, alkalisch gemacht und mit

1) Bericht d. d. chem. Gesellsch. XXIV, $2: 5$ n. Diss. v. Magalbaes. 
Chloroform ausgeschüttelt. Letzteres wurde abdestilliert und der Rückstand nach erfolgtem Lösen in salzsäurehaltigem Wasser mit Platinchlorid versetzt, die gelbe Fällung in salzsäurehaltigem Wasser gelöst, und die Lösung der langsamen Verdunstung anheimgestellt.

$$
\begin{aligned}
& \text { Isobutyl - Cytisin-Platinchlorid. } \\
& \mathrm{C}^{11} \mathrm{H}^{18}\left(\mathrm{C}^{4} \mathrm{H}^{9}\right) \mathrm{N}^{2} \mathrm{O} \cdot \mathrm{H}^{2} \mathrm{PtCl}^{\theta}+1 \frac{1}{2} \mathrm{H}^{2} \mathrm{O} .
\end{aligned}
$$

Anfänglich krystallisierte ein dunkelrotes, durchsichtiges, nadelartiges Doppelsalz aus, welches, als die Abscheidung der charakteristischen Krystalle des Monocytisinplatinsalzes begann, noch rechtzeitig ausgelesen werden konnte. Dieses Salz beginnt bei $230^{\circ}$ braun zu werden und zersetzt sich wenig über $250^{\circ}$.

$0,2186 \mathrm{~g}$ verloren bei $100^{\circ}$ getrocknet $0,0084 \mathrm{~g} \mathrm{H} \mathrm{H}^{2} \mathrm{O}=3,84 \frac{\sigma}{\%} ; 0,2102 \mathrm{~g}$ hinterliessen beim Glühen $0,0620 \mathrm{Pt}=29,49 \%$.

Gefunden:

$\mathrm{H}^{2} \mathrm{O} . \quad 3,84 \underset{\%}{\%}$

Pt. $29,47 \%$
Berechnet für Isobutyl-Cytisinplatinchlorid $\mathrm{C}^{11} \mathrm{H}^{18}\left(\mathrm{C}^{4} \mathrm{H}^{9}\right) \mathrm{N}^{2} \mathrm{O} \cdot \mathrm{H}^{2} \mathrm{PtCl}^{6}+1 \frac{1}{2} \mathrm{H}^{2} \mathrm{O}$ :

$3,95 \%$

für $\mathrm{C}^{11} \mathrm{H}^{13}\left(\mathrm{C}^{4} \mathrm{H}^{9}\right) \mathrm{N}^{2} \mathrm{O} \cdot \mathrm{H}^{2} \mathrm{PtCl}$ : $29,67 \%$.

Diese analytischen Daten beweisen, dass das alkoholische Alkali in der That die Alkylierung mit kohlenstoffreicherem Alkyljodid in diesem Falle günstig beeinflusst hatte. Indessen war die Ausbeute (aus 2,0 Cytisin waren nur etwa $0,25 \mathrm{~g}$ Platindoppelsalz erhalten worden) doch eine solch geringe, dass ich auf eine Vergleichung dieses Abkömmlings des Cytisins mit dem Anagyrin und seinen Salzen zunächst Verzicht leisten musste.

Einwirkung von tertiärem Butylchlorid auf Cytisin.

Der Versuch wurde mit 3,0 Cytisin und äberschüssigem tertiärem Butylchlorid in der Druckflasche vorgenommen, und die Dauer des Frhitzens auf drei Stunden bemessen. Der Inhalt war nach dieser Zeit deutlich in zwei Schichten getrennt, eine untere dunkelbraune, zähe, schmierige und eine darüber stehende rotbraune leichtbewegliche. Letztere wurde abgegossen; sie hinterliess nach dem Eindunsten einen geringen gelblich-braunen Rïckstand, der in salzsäurehaltigem Wasser gelöst und mit Platinchlorid versetzt wurde. Es trat hierbei Reduktion ein. Doch kamen aus dem Filtrate alsbald gelbe glänzende Krystalle zur Abscheidung.

$0,3494 \mathrm{~g}$ verloren bei $100^{0}$ getrocknet $0,0252 \mathrm{~g} \mathrm{H} \mathrm{H}^{2} \mathrm{O}=7,21 \% ; 0,3242 \mathrm{~g}$ des getrockneten Salzes lieferten 0,1056 g Pt $=32,57 \%$. 
Gefunden:

$\mathrm{H}^{2} \mathrm{O} . \quad 7,21 \%$

Pt. $\quad 32,57 \%$
Berechnet für $\mathrm{C}^{4} \mathrm{H}^{14} \mathrm{~N}^{2} \mathrm{O} \cdot \mathrm{H}^{2} \mathrm{Pt} \mathrm{Cl}^{6}+21 / 2 \mathrm{H}^{2} \mathrm{O}$ :

$$
6,98 \%
$$$$
\text { für } \mathrm{C}^{11} \mathrm{H}^{14} \mathrm{~N}^{2} \mathrm{O} \cdot \mathrm{H}^{2} \mathrm{PtCl}^{6} \text { : }
$$

$32,44 \%$.

Nach mehrtägigem Stehen war der schmierige Inhalt der Druckflasche teilweise erstarrt. Er wurde mit salzsäurehaltigem Wasser aufgenommen, die Lösung gelinde erwärmt, um die letzten Spuren anhaftenden tertiären Butylchlorids zu entfernen, alsdann in üblicher Weise in das Platinsalz verwandelt. Da hierbei Reduktion eintrat, wurde das Platin mit Schwefelwasserstoff zur Reinigung ausgefällt, und das schwefelwasserstofffreie Filtrat von neuem mit Platinchlorid versetzt. Nach längerem Stehen schieden sich hellgelbe, sternförmig gruppierte Nadeln aus, welche, ohne vorher zu schmelzen, sich beim Erhitzen zersetzten.

$0,2562 \mathrm{~g}$ verloren bei $100^{\circ}$ getrocknet $0,0182 \mathrm{~g} \mathrm{H} \mathrm{H}^{2} \mathrm{O}=7,10 \% ; 0,2380 \mathrm{~g}$ des getrockneten Salzes hinterliessen $0,0774 \mathrm{~g} \mathrm{Pt}=32,52 \%$.

Gefunden:

$\mathrm{H}^{2} \mathrm{O} . \quad 7,10 \%$

Pt. $32,52 \%$
Berechnet für Monocytisinplatinchlorid $\mathrm{C}^{11} \mathrm{H}^{14} \mathrm{~N}^{2} \mathrm{O} \cdot \mathrm{H}^{2} \mathrm{Pt} \mathrm{Cl}^{6}+2 \frac{1}{2} \mathrm{H}^{2} \mathrm{O}$;

$$
6,98 \%
$$

für $\mathrm{C}^{11} \mathrm{H}^{14} \mathrm{~N}^{2} \mathrm{O} \cdot \mathrm{H}^{2} \mathrm{PtCl}^{6}$;

$$
32,44 \% \text {. }
$$

Aus den Mutterlaugen krystallisierte ein dunkelgoldbraun gefärbtes durchsichtiges Salz ans, welches indessen, wie die unten stehende Analyse beweist, ebenfalls nur Monocytisinplatinchlorid darstellte.

$0,2258 \mathrm{~g}$ verloren bei $100^{\circ}$ getrocknet $0,0166 \mathrm{~g} \mathrm{H} 2 \mathrm{O}=7,35 \%$ und lieferten $0,0682 \mathrm{~g} \mathrm{Pt}=$ (für wasserfreies Salz) $32,60 \%$.

Einwirkung von normalem Butyljodid auf Cytisin.

Der Versuch wurde analog dem letztbeschriebenen ausgeführt. Der Inhalt der Druckflasche war nach dreistiundigem Erhitzen in dem Dampfbade grösstenteils fest geworden. Die durch Umkrystallisation gewonnenen gelblichen Krystalle schmolzen noch nicht bei $250^{\circ}$.

$0,2298 \mathrm{~g}$ verloren 6 Stunden bei $100^{\circ}$ getrocknet $0,0004 \mathrm{~g} \mathrm{H}^{2} \mathrm{O}$; sie ergaben $0,1499 \mathrm{~g} \mathrm{AgJ}=35,3 \% \mathrm{~J}$.

Es berechnen sich für

$$
\begin{array}{ll}
\text { jodw. Butylcytisin: } & 33,95 \% \mathrm{~J} \text { und für } \\
\text { jodw. Cytisin: } & 39,93 \% \mathrm{~J} \text {. }
\end{array}
$$

Das Filtrat vorstehender Jodbestimmung wurde von überschüssigem Silbernitrat durch Salzsäure befreit und zur Darstellung eines Goldsalzes benutzt. Der entstandene amorphe gelbe Niederschlag schied sich nach dem Auflösen in salzsäurehaltigem Wasser nur in braun- 
gefärbten klebrigen Tropfen aus. Zur Reinigung wurde aus der heissen Lösung derselben das Gold mit Schwefelwasserstoff ausgefällt und versucht, aus dem schwefelwasserstofffreien Filtrat von neuem ein Goldsalz darzustellen. Der umkrystallisierte Niederschlag stellte die für das Cytisingoldchlorid charakteristischen, rotbraunen, hakenförmig gekrümmten Nadeln dar, welche wie dieses bei $214-215^{\circ}$ schmolzen. Dass der Jodgehalt des Reaktionsproduktes von mir zu niedrig gefunden wurde, glaube ich auf die Beimengung harziger Verunreinigungen zurück fübren zu müssen, zumal hierfür auch das eigentümliche Verhalten des Filtrates dieser Jodbestimmung zu sprechen scheint, doch ist auch die Beimischung eines butylierten Produktes vielleicht nicht ganz ausgeschlossen.

Die von dem jodwasserstoffsauren Cytisin getrennte braune Flüssigkeit wurde bei gelinder Wärme eingedunstet und mit salzsäurehaltigem Wasser aufgenommen. Die sich abscheidenden harzigen Flocken wurden abfiltriert, das Filtrat alkalisch gemacht und mit Chloroform ausgeschüittelt. Der nach dem Abdestillieren des letzteren zurückgebliebene gelblich-braune Firnis diente zur Darstellung eines Platindoppelsalzes in der üblichen Weise. Die nach mehrmaliger Umkrystallisation erhaltenen gelben glänzenden Krystalle konnten leicht mit Monocytisinplatinchlorid identifiziert werden.

Einwirkung von sekundärem Butyljodid auf Cytisin.

Der Versuch wurde analog den früheren ausgeführt. Das Reaktionsprodukt, welches nur geringe Mengen eines weisslich-grauen Pulvers abgesetzt hatte, schied auch nach Zusatz von absolutem Alkohol und mehrtägigem Stehen nur noch wenige Krystalle ab, welche sich indessen durch Ueberschichten der Flüssigkeit mit Aether weiter noch vermehrten. Das Ausgeschiedene wurde schliesslich gesammelt und aus absolutem Alkohol umkrystallisiert. Die erhaltenen sternförmig gruppierten, weingelben Nadeln schmolzen noch nicht bei $250^{\circ}$.

0,3078 $\mathrm{g}$ des längere Zeit im Exsiccator aufbewahrten Krystallpulvers verloren bei $100^{\circ}$ getrocknet nichts an Gewicht und lieferten 0,2272 $\mathrm{g} \mathrm{Ag} \mathrm{J}=$ 0,1227 g $\mathbf{J}=39,86 \%$.

Gefunden:

J. $39,86 \%$
Berechnet für jodw. Cytisin $\mathrm{C}^{11} \mathrm{H}^{14} \mathrm{~N}^{2} \mathrm{O} \cdot \mathrm{HJ}$ : $39,93 \%$.

Zur weiteren Bestätigung dieses Befundes wurde das Filtrat obiger Jodbestimmung in das Goldsalz übergeführt, und die durch Umkrystallisation gewonnenen, $214^{0}-215^{\circ}$ schmelzenden rotbrauren Nadeln analysiert. 
$0,2622 \mathrm{~g}$ verloren bei $100^{\circ}$ getrocknet nichts an Gewicht und lieferten $0,0976 \mathrm{~g} \mathrm{Au}=37,22 \%$

Gefunden: Berechnet für Cytisingoldchlorid $\mathrm{C}^{11} \mathrm{H}^{14} \mathrm{~N}^{2} \mathrm{O} \cdot \mathrm{HAuCl}^{4}$ :

Au. $37,22 \%$ $37,11 \%$

Die Mutterlauge des festen Reaktionsproduktes wurde vorsichtig eingedunstet, mit Wasser aufgenommen, mit Kalilauge alkalisch gemacht und nun mit Chloroform ausgeschüttelt.

Sekundäres Butyl-Cytisinplatinchlorid.

Obige Chloroformlösung lieferte nach dem Abdestillieren des Lösungsmittels einen hellgelben, öligen Rückstand. Er wurde durch Erwärmen in salzsäurehaltigem Wasser gelöst, und nunmehr versucht. aus ihm ein Platinsalz darzustellen. Die durch wiederholte Umkrystallisation gewonnenen harten, rotgelben Krystalle sind sowohl in heissem Wasser als auch in salzsäurehaltigem heissen Wasser schwer löslich, leichter jedoch bei Gegenwart von Alkohol. Beim Erhitzen zersetzte sich das Salz bereits bei $180^{\circ}$, obne vorher zu schmelzen.

$0,2304 \mathrm{~g}$ verloren bei $100^{\circ}$ getrocknet $0,0168 \mathrm{~g} \mathrm{H}^{2} \mathrm{O}=7,29 \% ; 0,2136 \mathrm{~g}$ wasserfreie Substanz lieferten $0,0620 \mathrm{~g} \mathrm{Pt}=29,02 \%$.

Gefunden:

$\mathrm{H}^{2} \mathrm{O} . \quad 7,29 \stackrel{\circ}{\circ}$

Pt. $\quad 29,02 \%$
Berechnet für sek. Butyl-Cytisinplatinchlorid $\mathrm{C}^{11} \mathrm{H}^{13}\left(\mathrm{C}^{4} \mathrm{II}^{9}\right) \mathrm{N}^{2} \mathrm{O} \cdot \mathrm{H}^{2} \mathrm{PtCl}^{6}+3 \mathrm{H}^{2} \mathrm{O}$ : $7,61 \%$ für $\mathrm{C}^{11} \mathrm{H}^{13}\left(\mathrm{C}^{4} \mathrm{H}^{9}\right) \mathrm{N}^{2} \mathrm{O} \cdot \mathrm{H}^{2} \mathrm{PtCl}^{6}$ : $29,67 \%$.

Elementaranalyse:

$0,1992 \mathrm{~g}$ verloren bei $100^{\circ}$ getrocknet $0,0146 \mathrm{~g} \mathrm{H} \mathrm{H}^{2} \mathrm{O}=7,35 \% ; 0,1846 \mathrm{~g}$ des getrockneten Salzes lieferten $0,1898 \mathrm{~g} \mathrm{CO} \mathrm{CO}^{2}=0,05176 \mathrm{~g} \mathrm{C}=28,03 \%$ und $0,0604 \mathrm{~g} \mathrm{H}^{2} \mathrm{O}=0,00671 \mathrm{~g} \mathrm{H} \mathrm{3,63 \%}$.

Gefunden: $\quad$ Berechnet für $\mathrm{C}^{11} \mathrm{H}^{13}\left(\mathrm{C}^{4} \mathrm{H}^{9}\right) \mathrm{N}^{2} \mathrm{O} \cdot \mathrm{H}^{2} \mathrm{PtCl}^{6}+3 \mathrm{H}^{2} \mathrm{O}$ : H2 O $\quad 7,85 \%$

C. $28,03 \%$

H. 3,63 , $7,61 \%$ für $\mathrm{C}^{11} \mathrm{H}^{19}\left(\mathrm{C}^{4} \mathrm{H}^{9}\right) \mathrm{N}^{2} \mathrm{O} \cdot \mathrm{H}^{2} \mathrm{Pt} \mathrm{Cl}^{6}$ : $27,45 \%$ 3,66 ,

Eine weitere Bestätigung der erfolgten Butylierung musste dahingestellt bleiben, weil mein ganzes Material mit Ausnahme weniger Belegkrystalle durch die ausgeführten Bestimmungen aufgebraucht worden war. Ein Versuch, das Einwirkungsprodukt von sek. Butyljodid auf Cytisin in grosser Menge unter Zusatz des entsprechenden Alkohols darzustellen, schlug gänzlich fehl.

Schliesslich wiederholte ich die diesbezügliche Untersuchung, indem ich in der schon oft skizzierten Weise sek. Butyljodid auf Cytisin ohne Zusatz des korrespondierenden Alkohols zur Einwirkung 
brachte. Das Reaktionsprodukt. wurde in der üblichen Weise untersucht. Auch diesmal war in der Hauptsache jedoch nur jodwasserstoffsaures Cytisin gebildet worden, welches sich an den Wandungen der benutzten Druckflasche als gelblich-weisses Pulver abgesetzt hatte. Der nicht feste Anteil wurde nach dem Verdunsten des überschüssigen Butyljodids alkalisch gemacht und mit Chloroform ausgeschüttelt. Der endlich nach dem Abdestillieren des letzteren zurückgebliebene gelbliche Firnis diente zur Darstellung einer Platinverbindung. Aus den ersten Lösungen des mit Platinchlorid erzielten Niederschlages kamen sehr schöne, vierseitige Tafeln zur Abscheidung, während die Mutterlaugen dieser Krystallisationen bei weiterem Eindunsten nur die für Monocytisinplatinchlorid charakteristischen grossen Nadeln lieterten.

\section{Sekundäres Butyleytisinplatinchlorid.}

Die Gesamtmenge der gewonnenen Tafeln betrug etwa $0,8 \mathrm{~g}$ (aus 5,0 Cytisin).

1. $0,2640 \mathrm{~g}$ verloren bei $100-120^{\circ}$ getrocknet $0,0102 \mathrm{~g} \mathrm{H}^{2} \mathrm{O}=3,91 \%$; $0,2502 \mathrm{~g}$ des getrockneten Salzes hinterliessen $0,0736 \mathrm{~g} \mathrm{Pt}=29,42 \%$.

2. $0,2854 \mathrm{~g}$ verloren bei $100-120^{\circ}$ getrocknet $0,0112 \mathrm{~g} \mathrm{H}^{2} \mathrm{O}=3,92 \%$; $0,2742 \mathrm{~g}$ des getrockneten Salzes lieferten 0,0812 g Pt $=29,61 \%$.

Gefunden:

$\mathrm{H}^{2} \mathrm{O} . \quad 3,91 \%$ 3,92 ,

Pt. 29,42 , 29,61 ,

Berechnet für sek. Butyl-Cytisinplatinchlorid $\mathrm{C}^{11} \mathrm{H}^{18}\left(\mathrm{C}^{4} \mathrm{H}^{9}\right) \mathrm{N}^{2} \mathrm{O} \cdot \mathrm{H}^{2} \mathrm{PtCl}^{6}+11 / 2 \mathrm{H}^{2} \mathrm{O}$ :

$$
3,95 \%
$$

für $\mathrm{C}^{11} \mathrm{H}^{18}\left(\mathrm{C}^{4} \mathrm{H}^{9}\right) \mathrm{N}^{2} \mathrm{O} \cdot \mathrm{H}^{2} \mathrm{PtCl}^{6}$ :

$$
29,67 \% \text {. }
$$

Diese Daten beweisen, dass bei obigem Versuche in geringem Umfange Butylierung erfolgt ist. Das analysierte Platinsalz ist von dem früher beschriebenen Salze des sek. Butylcytisins nicht nur hinsichtlich der Krystallform, sondern auch wesentlich durch den Krystallwassergehalt unterschieden. Das mit 1/1/2 Mol. krystallisierende Platinsalz lehnt sich sowohl, was den Wassergehalt, als auch dessen Abgabe bei höherer Temperatur anbetrifft, sehr an das bereits früher beschriebene, mit 1 $1 \frac{1}{2}$ Mol. krystallisierende Salz des Anagyrins an. In der äusseren Form und Gestalt lassen sich jedoch keine Aehnlichkeiten nachweisen. Nach Partheil ${ }^{1}$ ) soll zwar das Anagyrinplatinchlorid ebenfalls gelegentlich in Form von Täfelchen zur Abscheidung gelangen können, indessen habe ich niemals Gelegenheit gehabt, dieses bei meinen Untersuchungen zu beobachten. Die Frage, ob möglicherweise zwischen

1) Apoth.-Ztg. 1895, No. 103.

Arch. d. Pharm. CCXXXVIII. Bds. 3. Heft. 
dem Platinsalz des Anagyrins und dem des sek. Butylcytisins Identität vorwaltet, kann vorläufig nicht erörtert werden.

Die bei obigen Butylierungsversuchen gesammelten Erfahrungen kurz zusammengefasst lehren, dass die Anlagerung kohlenstoffreicherer Halogenalkyle an das Cytisin mit grossen Schwierigkeiten verknüpft ist. Die erfolgte teilweise Alkylierung konnte nur bei den Versuchen mit Isobutyljodid und mit sek. Butyljodid einwandsfrei bewiesen werden, während bei den mit tert. Butylchlorid und norm. Butyljodid vorgenommenen wahrscheinlich überhaupt keine Reaktion in dem gewünschten Sinne stattfand. Vorläufig fehlt eine Methode, welche zu besseren Erfolgen in dieser Richtung führt, und wird daher die Möglichkeit einer Partialsynthese des Anagyrins aus Cytisin hierdurch sehr in Frage gestellt. Dennoch gedenke ich in der Folge weitere diesbezügliche Untersuchungen vorzunehmen.

\section{Einwirkung von Isobutyljodid und sekundärem Butyljodid auf Methylcytisin.}

Bekanntlich addieren einige tertiäre Basen leichter Halogenalkyle als sekundäre. Da das als tertiäre Base fungierende Methylcytisin ohne Schwierigkeiten in reinem Zustande darstellbar ist, wandte ich meine weiteren Studien einigen Untersuchungen zu, welche das Verhalten des Methylcytisins gegen verschiedene Butyljodide prüfen und feststellen sollten. Wenn die gewünschte Einwirkung thatsächlich erfolgte, so musste aller Wahrscheinlichkeit nach eine mit dem früher beschriebenen Anagyrinmethyljodid identische oder doch isomere Verbindung entstehen, und sich ein neues Feld interessanter Untersuchungen erschliessen lassen.

Nach Partheil's Angaben ${ }^{1}$ ) wurde eine grössere Menge Methylcytisin dargestellt, und durch eine Analyse des charakteristischen Platinsalzes die Reinheit des ersteren dargethan.

Es wurden folgende Versuche ausgführt:

a) Methylcytisin wurde mit überschüssigem Is obutyljodid

1. 3 Stunden lang in der Druckflasche der Wasserbadwärme ausgesetzt;

2. 3 Stunden lang in zugeschmolzenem Rohre bei $120^{\circ}$ erhitzt;

3. wie 1, jedoch erst nach Zusatz von Isobutylalkohol;

4. wie 1., jedoch erst nach Zusatz von Amylalkohol;

5. in verschlossener Flasche mehrere Wochen hindurch bei Zimmertemperatur stehen gelassen;

6. wie 5., jedoch nach Zusatz von wenig Chloroform.

1) Dieses Archiv 1892. 
b) Methyleytisin wurde ferner mit überschüssigem sekundärem Butyljodid behandelt, wie unter 1-6 angegeben ist.

Sämtliche Versuche verliefen negativ. Neben oft beträchlicher Verharzung des Methylcytisins konnte durch Ueberführung der Einwirkungsprodukte in das jeweilige Platinsalz und durch dessen Analyse stets nur unverändertes Methylcytisin nachgewiesen werden.

Bei dem Glühen dieser Platinsalze wurden folgende Werte für $\mathrm{Pt}$ in Prozenten gefunden:

Versuche a: 1. 31,56 ; 2. 31,$73 ; 3$. 31,$78 ; 4$. 31,$65 ; 5.31,42 ; 6.31,62$. Versuche b: 1. 31,79 ; 2. 31,85 ; 3. 31,$78 ; 4$. 31,$90 ; 5.31,88$; 6. 31,93 .

Es berechnet sich für wasserfreies Methylcytisinplatinchlorid $\mathrm{C}^{12} \mathrm{H}^{16} \mathrm{~N}^{2} \mathrm{O} \cdot \mathrm{H}^{2} \mathrm{Pt} \mathrm{Cl}^{6}$ :

Pt. $31,70 \underset{\%}{o}$.

\section{Ueber das Anagyrin.}

Von Dr. Max Klostermann.

Das zu meinen Untersuchungen ${ }^{1}$ ) verwendete Anagyrin war nach dem in der vorstehenden Arbeit skizzierten Verfahren von Partheil und Spasski bereitet worden. Dasselbe bildete eine blassgelbliche, firnisartige Masse, die bisher nicht zur Krystallisation gebracht werden konnte. Die Formel $\mathrm{C}_{15} \mathrm{H}_{22} \mathrm{~N}_{2} \mathrm{O}$, welche für diese Base von mir acceptiert wurde, konnte daher nur durch die Daten begründet werden, die bei der Analyse der Salze, Doppelsalze und der sonstigen Abkömmnlinge erzielt wurden.

Das Anagyrin erwies sich als leicht löslich in Wasser, Chloroform, Essigäther, absolutem und verdünntem Alkohol, sowie in wasserhaltigem Aether, als weniger leicht löslich dagegen in wasserfreiem Aether und in Ligroin, als schwer losslich in Benzol. Es wurde vergeblich versucht dieses Alkaloid aus Wasser, Chloroform, Aether und Alkohol zur Krystallisation zu bringen. In siedendem Ligroïn löste es sich zwar auf, jedoch schied es sich beim Erkalten in öligen Tropfen wieder aus. Zusatz von absolutem Alkohol verhinderte zwar diese ölige Ausscheidung, veranlasste aber bei der freiwilligen Verdunstung dieser Lösung keine Krystallbildung.

Beim Erwärmen mit Essigäther spaltete Anagyrin hieraus Essigsäure ab.

1) Inauguraldissertation Marburg 1898. 